\title{
Experimental investigation of the water entry and/or exit of axisymmetric bodies
}

\author{
Breton Thibaut ${ }^{1}$, Tassin Alan ${ }^{1}$, Jacques N. ${ }^{2}$ \\ 1 Ifremer, RDT, F-29280 Plouzané, France \\ 2 ENSTA Bretagne, UMR CNRS 6027, IRDL, 29806 Brest CEDEX 09, France
}

\begin{abstract}
:
This paper presents an experimental investigation of the evolution of the wetted surface and of the hydrodynamic force during the water exit of a body initially floating at the water surface, and during combined water entry and exit. The evolution of the surface of contact between the body and the water is measured using transparent mock-ups and an LED edge-lighting system. This technique makes it possible to follow the evolution of the wetted surface during both the entry and exit phases with a highspeed video camera placed above the mock-up. The feasibility of the technique is shown for different axisymmetric bodies: a circular disc, a cone and a sphere. The evolution of the hydrodynamic force and of the radius of the wetted surface measured during the experiments are compared with theoretical results obtained with a combined Wagner-modified von Karman approach (Tassin et al. J. Fluids Struct., vol. 40, 2013, pp. 317-336), the linearized water exit model of Korobkin et al. (J. Fluids Struct., vol. 69, 2017a, pp. 16-33) and the small-time self-similar solution of Korobkin et al. (J. Engng Maths, vol. 102, 2017b, pp. 117-130).
\end{abstract}

Keywords : free-surface flows, wave-structure interactions 


\section{Introduction}

The water exit phenomenon designates the upward lifting of a body initially floating at the water surface or the lifting of a body which previously entered the water. One may speak of a combined water entry and exit event in the latter case. Studies on this hydrodynamic phenomenon are motivated by various marine applications such as wave impact on offshore platforms (Baarholm \& Faltinsen 2004) and on suspended structures (Sun \& Helmers 2015), or ship slamming (Kaplan 1987). The aeronautical industry has also to deal with water entry and exit to predict the hydrodynamic loads acting on the fuselage of an airplane during emergency landing (see Bensch et al. 2001; Tassin et al. 2013). It is also interesting to note that one of the first experimental studies on the water exit phenomenon was conducted by researchers in biomechanics in order to understand the cat lapping phenomenon (Reis et al. 2010).

Both water entry and water exit involve a rapid evolution of the surface of contact between the body and the liquid, which is also called the wetted surface. Following the definition commonly adopted in water entry problems (see Korobkin 2007), the contact surface designates the surface delimited by the line(s) where the free surface is vertical, $|x|=c(t)$ in the two-dimensional case illustrated in figure 1a. During an entry stage the contact surface is in expansion whereas the contact surface is contracting during an exit stage. In general, during the water entry and exit of a rigid body moving vertically, the water entry (resp. water exit) stage corresponds to the period during which the body moves downward (resp. upward). In the case of entry with deceleration and then

$\dagger$ Email address for correspondence: alan.tassin@ifremer.fr 


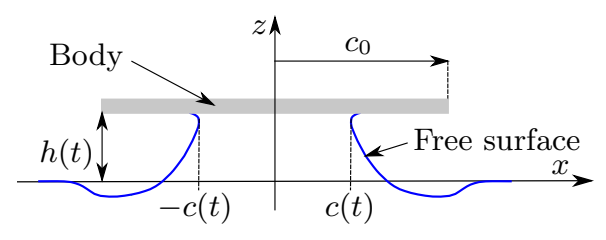

(a) Water exit of a flat plate

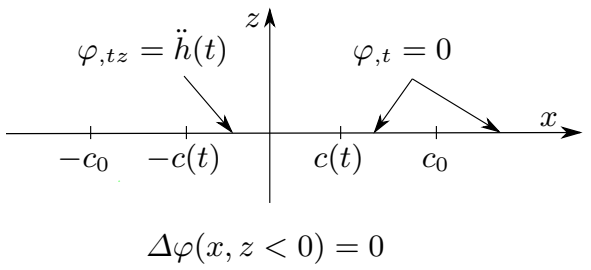

(b) Linearized mixed boundary value problem satisfied by the velocity potential $\varphi$ in Tassin et al. (2013) and Korobkin (2013)

Figure 1: Water exit of a flat plate and linearized water exit problem

exit, suction loads (hydrodynamic pressures being below the atmospheric pressure) arise during the entry stage and the hydrodynamic force remains negative (directed downward) during most of the exit stage. Also, when the body deceleration is constant, the maximum suction force occurs at the transition between the entry stage and the exit stage (Tassin et al. 2013). However, when dealing with the complex motion of highly deformable bodies, the notion of entry or exit is less obvious and we suggest to define the entry and exit stages based on the evolution of the contact point (which is not known a priori). Note that in the numerical simulations of Piro \& Maki (2013b) corresponding to the water entry and exit of a rigid wedge with separation occuring at the knuckle, the water starts separating from the surface of the body while the wedge is still going downward (see figure 7, right column, second row, in Piro \& Maki 2013b). As a consequence, the maximum suction force occurs before the wedge starts going up again (see figure 9b in Piro \& Maki 2013b). The suction load and the separation of the liquid from the body surface are two important features characterising the water exit phenomenon, but they can be observed in other multiphase problems. Indeed, suction and flow separation are observed during the oblique water entry of wedges and curved bodies at high horizontal speed (see Semenov \& Yoon 2009; Reinhard et al. 2012; Reinhard 2013). The magnitude of the suction loads can lead to cavitation on the fuselage of an aircraft (Iafrati \& Grizzi 2019). Flow separation also occurs during the vertical water entry of curved bodies (e.g. sphere, cylinder) when the entry speed is sufficiently large (Duez et al. 2008). These examples show that flow separation and suction are closely related. The water exit problem is a good case study in order to investigate the suction and flow separation phenomena. A better understanding of the water exit phenomenon would probably help understanding the other mentioned phenomena involving suction and flow separation.

Very few studies have been dedicated to the water exit problem in comparison to the extensive research on the water entry problem. Most of the investigations were so far based on analytical and numerical approaches. To the authors' knowledge, the first analytical model aiming at modelling the exit stage (subsequent to an entry stage) was proposed by Kaplan (1987). In this model, the entry stage was modelled with the original Wagner model (Wagner 1931, 1932) and the Wagner model was modified in order to make it compatible with the exit stage. This modification consisted in disregarding the "slamming term" and retaining only the "added mass" term in the Wagner model when calculating the hydrodynamic force during the exit stage. A similar approach was later 
used by Bensch et al. (2001) and Baarholm \& Faltinsen (2004). More recently, Tassin et al. (2013) and Korobkin (2013) showed that a similar result could be obtained by solving the linearized mixed boundary value problem described in figure $1 \mathrm{~b}$ (where $\varphi$ is the velocity potential) and by enforcing the Kutta-type condition $\varphi_{, t}\left(|x|=c(t)^{-}\right)=0$ at the contact point during the exit stage (with $\varphi_{, t}$ the time derivative of $\varphi$ ). A consistent analytical model of water exit shall predict accurately the evolution of the wetted surface and the hydrodynamic force (or pressure distribution). In Tassin et al. (2013), the contact point position is determined by a modified von Karman approach in which the boundary of the contact surface corresponds to the geometrical intersection between a horizontal plane of reference and the body surface. The altitude of the reference plane is set to the maximum altitude reached by the contact point during the entry stage (at the transition between entry and exit). The pressure is computed with the Modified Logvinovich Model (MLM), but the only terms which remain during the exit stage depend on the body acceleration (added mass effect). Despite the simplicity of the approach used for the prediction of the contact point position, the results obtained in terms of pressure distribution and hydrodynamic force are in rather good agreement with the reference numerical results during the beginning of the water exit stage (when the suction force is maximal). In the linearized model of Korobkin (2013), a more elaborated condition is used to predict the contact point position. This author assumes that the speed of contraction of the contact surface is proportional to the velocity of the particles located at the contact points. For a two-dimensional problem in the lower $x$ - $z$ half-plane $(z<0)$, the contact point condition reads: $\dot{c}(t)=\gamma \cdot \varphi_{, x}(|x|=c(t), z=0)$, with $\dot{c}(t)$ the time derivative of the half-width of the wetted surface and $\varphi_{, x}$ the horizontal gradient of the velocity potential (i.e. the horizontal velocity). Korobkin (2013) found that a value of $\gamma=2$ gave a very good prediction of the hydrodynamic force during almost the entire duration of the exit stage. However, the accuracy of the prediction of the contact surface with this value of $\gamma$ has not been assessed. Note that Korobkin's condition for the evolution of the contact point position was used earlier by Baarholm \& Faltinsen (2004) (with $\gamma=1$ ) to ensure the stability of their nonlinear potential flow simulations during the exit stage of a wave impact. It is indeed also difficult to accurately simulate numerically water exit problems, especially when the exit is subsequent to a water entry stage. Indeed, the numerical algorithms used in Computational Fluid Dynamics (CFD) are not all able to handle both the entry and the exit stage. For instance, Piro \& Maki (2013a) observed that the interface compression scheme, that allows to improve the accuracy of water entry simulations (with a multiphase CFD approach) leads to free-surface instabilities during water exit. To circumvent this problem, they proposed an adaptative interface compression algorithm in order to improve the simulation of the exit stage. In the finite element methods based on a single phase arbitrary Euler-Lagrange approach, it is common to use a penalty contact algorithm for the simulation of water impact problems (Tassin et al. 2012). However, this approach cannot properly handle the suction (i.e. the occurence of negative relative pressure). The same problem may occur in simulations based on the Smoothed Particle Hydrodynamics (SPH) method (Panciroli et al. 2012).

New experimental studies are necessary to improve our knowledge of the water exit phenomenon, to validate the numerical simulations and to help developing new analytical models. Indeed, to date, very few experimental results are available. Reis et al. (2010) conducted their experiments at very low scale on circular discs and without measuring the hydrodynamic force nor the evolution of the wetted surface. More recently, VegaMartínez et al. (2019) published experimental results on the water exit of a circular disc, 
but the elasticiy of the disc had a large effect on the results and the measurement of the wetted surface was restricted to the very beginning of the water exit $(c(t) / c(0)>0.9)$. Based on the above observations on the water exit stage, experimental results in terms of contact surface and hydrodynamic force are of prime interest. For this purpose, we conducted both water exit and combined water entry and exit experiments with axisymmetric bodies (a circular disc, a cone and a spherical cap). The choice of axisymmetric body shapes over two-dimensional bodies was made in order to avoid undesired three-dimensional effects that may appear with a "pseudo" two-dimensional experimental set-up. Indeed, with two-dimensional mock-ups, three-dimensional effects always take place at the extremities of the specimen (e.g. Shams et al. 2017). The axisymmetric problem also has the advantage to remain tractable with analytical approaches. Indeed, the analytical models of Tassin et al. (2013) and Korobkin (2013) are readily applicable to axisymmetric water exit problems. The different body-shapes considered in the present study make it possible to assess the effect of the shape on the water exit phenomenon.

One original aspect of our work is the use of transparent mock-ups and a LED edge-lighting device to track the contour of the contact surface (the contact line) during the experiments. We previously showed the feasibility of this technique for flat plates of different shapes (a circular disc and a square plate) in Tassin et al. (2017). This technique was proposed as an alternative to the bottom view approach used in previous water entry experiments (e.g. Halbout 2011), which we found to be inappropriate for the tracking of the contact line during the water exit of a circular disc. We now report on a detailed study on the water exit and combined water entry and exit of different three-dimensional mock-ups, hence demonstrating the applicability of the edge-lighting technique for three-dimensional bodies. More importantly, we show that is possible to track both the contact line and the jet front during the entry stage. The experiments have been carried out in a medium-scale wave tank with a dedicated metallic frame fixed on a 6 degree-of-freedom motion generator that allows an accurate control of the motion of the mock-ups. Moreover, with this new experimental set-up, it is possible to record simultaneously the evolution of the contact line and the hydrodynamic force. To validate the accuracy of the LED edge-lighting technique, each experiment has been realized with and without a draughtboard placed at the bottom of the wave tank. With this technique inspired from Scolan et al. (2006), the wetted surface corresponds to the region over which the draughtboard appears as undistorted (except for the distortion due to the shape of the body and to the perspective).

Through this experimental study, several aspects of the water exit phenomenon are studied:

- We investigate the influence of the body shape on the water exit problem. In particular, we compare the evolution of the wetted surface and of the hydrodynamic force for the three different shapes (circular disc, cone, sphere), but with the same initial wetted surface and the same experimental conditions.

- The effect of viscosity and surface tension is assessed by carrying out experiments in Froude similarity with the conical mock-up for different values of the initial wetted surface (by varying the initial penetration depth).

- The evolution of the wetted surface measured during a water exit experiment is compared to the evolution of the wetted surface measured during the exit stage of a combined water entry and exit experiment.

- The experimental results are compared with different theoretical models: the 
combined Wagner-modified von Karman model of Tassin et al. (2013), the linearized water exit model of Korobkin et al. $(2017 a)$ and the small-time self-similar solution of Korobkin et al. $(2017 b)$. For this purpose, an extension of the linearized model of Korobkin et al. $(2017 a)$ to the water exit of a rigid axisymmetric body with an arbitrary exit kinematics $(\ddot{h}(t) \geqslant 0)$ was implemented.

The article is organised as follows: section 2 describes the experimental set-up, section 3 explains the method used for the analysis of the measurements, section 4 presents the experimental results, section 5 discusses the effect of different parameters affecting water exit and presents comparisons with theroretical results, and finally the conclusions of our study are drawn in section 6. A detailed validation of the LED edge-lighting technique through comparisons with the draughtboard technique is also presented in appendix A.

\section{Experimental set-up}

The experiments were conducted in the wave and current flume of IFREMER in Boulogne-sur-mer (France). Our investigation of the water exit phenomenon motivated the development of a dedicated experimental set-up which is described in figure 2 . This set-up is composed of a metallic frame, a transparent mock-up and a 6-degree-of-freedom motion generator (see figure 2a). The motion generator (hexapod) is used to adjust the inclination of the set-up and to move the set-up vertically during the experiments. The entire set-up is placed above a medium scale wave tank $(2 \mathrm{~m}$ deep, $4 \mathrm{~m}$ wide and $20 \mathrm{~m}$ long) and half-way from the sidewalls of the tank. The evolution of the contact line is recorded by an onboard high-speed video camera (Photron Fastcam Mini AX50) looking downward and fixed to the metallic frame above the center of the mock-up. As the camera follows the motion of the mock-up, the distance between the camera and the mock-up is constant during the experiments. The images presented in this paper were recorded at a frame rate of $1000 \mathrm{fps}$ and using a Nikon $20 \mathrm{~mm}$ F2.8 lens. The hydrodynamic force was recorded using 3 piezolelectric load cells (Kistler 9331B) connected to a dynamic (and quasi-static) charge amplifier (Kistler LabAmp type 5167A40FK). The load cells were placed between the mock-up and the metallic frame in order to minimise the mass of the elements below the force sensors and which may induce an inertial force component during the experiments. Although we tried to place the force sensors as close as possible to the mock-up and to minimize the mass of the mock-up, inertia forces are unavoidable during water exit experiments because the velocity of the mock-up is not constant. In order to extract the hydrodynamic force component from the total force measured by the sensors, all the experiments were carried out both in air and water with identical motions. This way, it was possible to substract the inertia force component measured in air (without touching the water) from the force measured during the water entry and/or exit experiments. Note that in pure water entry experiments, inertia effects can be mitigated by enforcing a constant water entry velocity, but it is also common to compensate the variation of velocity during water entry experiments (see El Malki Alaoui et al. 2012).

\subsection{Description of the transparent mock-ups}

The technique employed for the visualization of the wetted surface during the experiments is based on the use of transpartent mock-ups and a LED edge-lighting technique. Three transparent shells of different shapes were built: a circular disc, a cone and a hemisphere. Note that each mock-up corresponds to an axisymmetric transparent shell 


Motion generator
Accelerometer
High-speed video
camera
Force sensors
Transparent mock-
up

(a) Sketch of the experimental set-up (dimensions are given in $\mathrm{mm}$ )
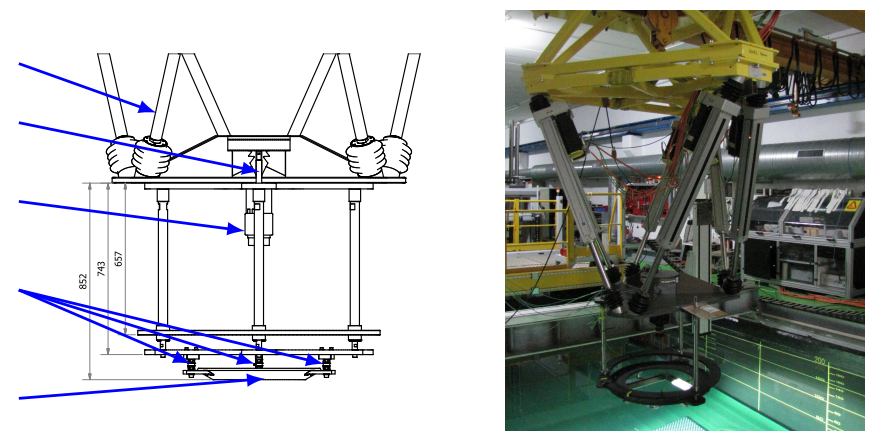

(b) Photograph of the experimental set-up

Figure 2: Experimental set-up

which was manufactured in a block of PMMA and finely polished. An array of highpower LEDs was mounted at the periphery of each mock-up in order to diffuse light in the material. The LED array was covered of aluminium cellotape in order to maintain it in position and to direct the light toward the mock-up. A vertical cross-section and a picture of each mock-up is displayed in figure 3. In figure 3a, one can see that the circular disc is composed of a $40 \mathrm{~cm}$ central region which is $15 \mathrm{~mm}$ thick and a sloped region at the periphery of the mock-up which is $11 \mathrm{~mm}$ thick. The disc was continued obliquely with a slope of $30^{\circ}$ in order to prevent the LEDs from being in contact with the water. The conical mock-up described in figure $3 \mathrm{c}$ is composed of a central part with a deadrise angle of $15^{\circ}$ and a thickness of $15 \mathrm{~mm}$. For the same reason as in the case of the disc, the conical mock-up was continued obliquely with a $30^{\circ}$ tilted edge of $12 \mathrm{~mm}$ thickness. The spherical mock-up shown in figures $3 \mathrm{e}$ and $3 \mathrm{f}$ corresponds to a truncated hemispherical shell with an external radius of curvature of $353.6 \mathrm{~mm}$ and a thickness of $15 \mathrm{~mm}$. In contrast to the other mock-ups, the spherical mock-up was continued tangentially by 40 $\mathrm{mm}$. The extrapolated sides of each mock-up were covered with an aluminum cellotape because of the bad quality of the polishing in these regions. The cellotape acted as a light guide which increased the diffusion of light in the shell and limited the direct lighting of the free surface by the diffusive peripheral regions of the mock-ups. Note that in Tassin et al. (2017), it was not necessary to cover the periphery of the mock-up by aluminuim cellotape thanks to the good quality of the polishing. The edge of the mock-ups in contact with the LEDs was roughened to homogenize the light diffusion in the material.

\subsection{Compensation of optical distortion}

In the case of the three-dimensional mock-ups (cone, sphere), it was necessary to compensate for the optical distorsion due to the perspective effects (mainly). In order to do so, we manufactured calibration parts over which the projection of a regular grid was printed. These parts were placed in contact with the lower face of the mock-up (see figure 4) and an image of the grids seen through the mock-up was recorded with the video camera before the series of experiments (see figure $5 \mathrm{a}$ ). The localisation of the intersections between the vertical and horizontal lines in the calibration images was performed with an in-house algorithm developped for the detection of the intersections. A comparison of the measured and theoretical positions of the grid intersections in the case of the cone is shown in figure 5b. The intersection-point positions were then used to identify a $8^{t h}$-order polynomial correction model (see Tang et al. 2017) of the following 
LED array

(covered by aluminium cellotape)

$15^{\circ}$

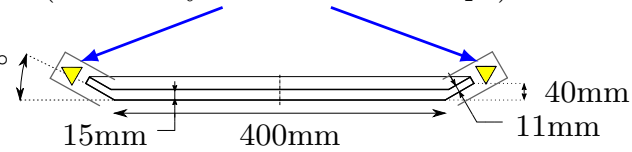

(a) Description of the circular disc

LED array

(covered by aluminium cellotape)

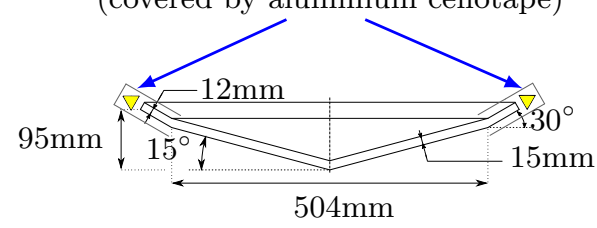

(c) Description of the circular cone

LED array

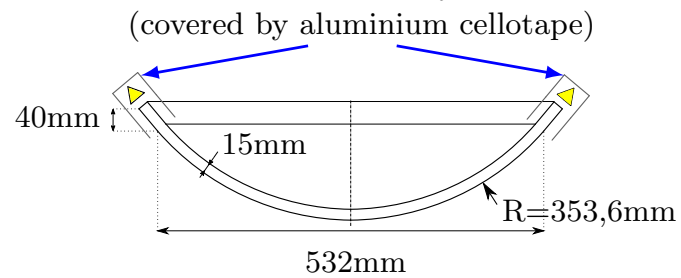

(e) Description of the sphere

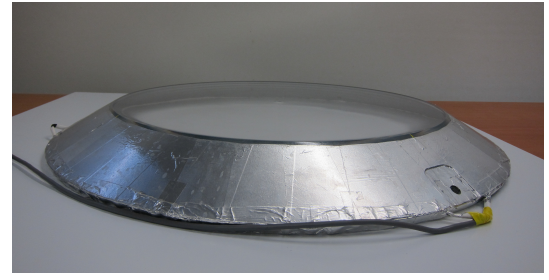

(b) Photograph of the disc

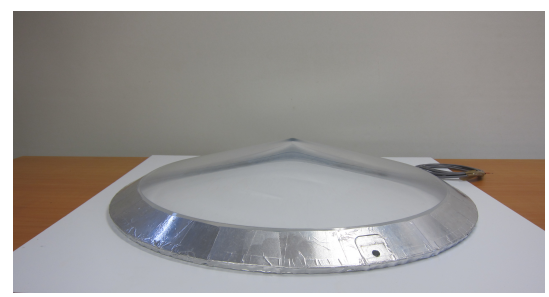

(d) Photograph of the cone

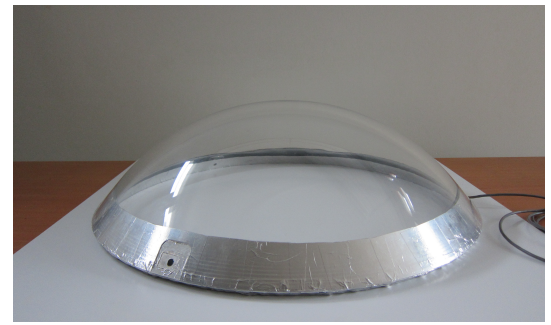

(f) Photograph of the sphere

Figure 3: Description of the different mock-ups used in the experiments

form:

$$
\left\{\begin{array}{l}
x_{2}=a_{1} x_{1}^{8}+a_{2} x_{1}^{7} y_{1}+\ldots+a_{44} y_{1}+a_{45} \\
y_{2}=b_{1} x_{1}^{8}+b_{2} x_{1}^{7} y_{1}+\ldots+b_{44} y_{1}+b_{45}
\end{array}\right.
$$

where $\left(x_{1}, y_{1}\right)$ are the positions of the pixels as observed by the camera and $\left(x_{2}, y_{2}\right)$ are the corrected positions of the points in the $x-y$ plane. The value of the parameters of the correction model $\left(a_{1}, \ldots, a_{45}, b_{1}, \ldots, b_{45}\right)$ were adjusted using an optimization algorithm based on the minimization of the distance between the measured and theoretical position of the calibration grid intersections. The absolute residual error maps after calibration for the cone and for the sphere are displayed in figures $5 \mathrm{c}$ and $5 \mathrm{~d}$, respectively. One can see in figures $5 \mathrm{c}$ and $5 \mathrm{~d}$ that the average residual error after calibration is lower than one millimeter, except near the centre of the cone where the error reaches $2 \mathrm{~mm}$. The higher error near the centre of the cone can be explained by the presence of manufacturing imperfections at the centre of the cone which affect the accuracy of the intersection detection algorithm. We verified a posteriori that the distortion was negligible in the case of the circular disc (a calibration part was also manufactured for this mock-up). 
Spherical mock-up

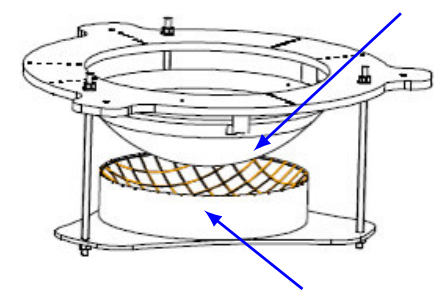

Calibration part

Figure 4: Calibration part manufactured for the sphere

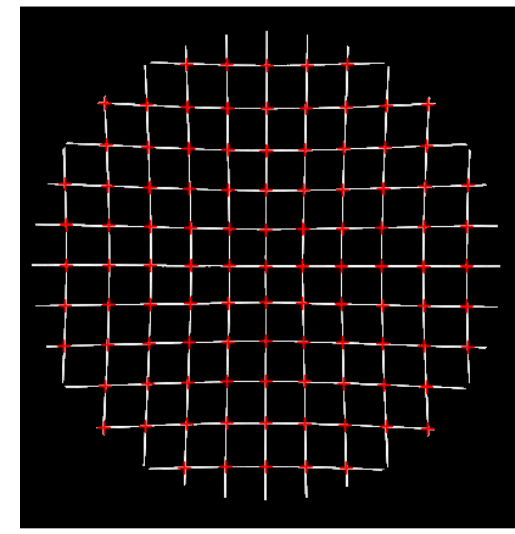

(a) Calibration grid seen through the cone with reversed contrast

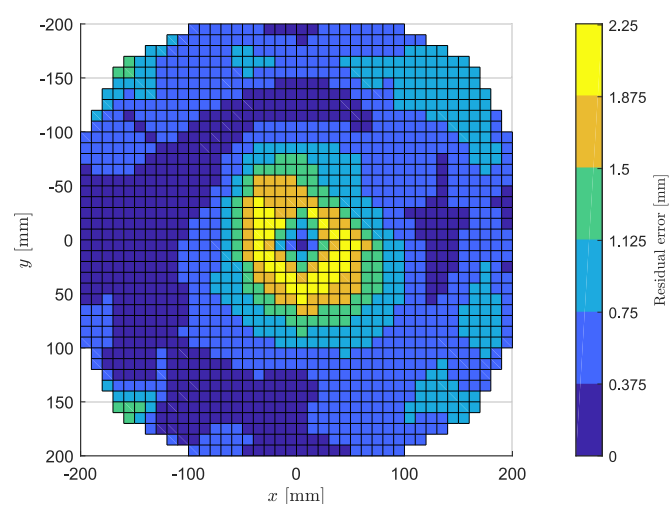

(c) Residual error after calibration of the cone

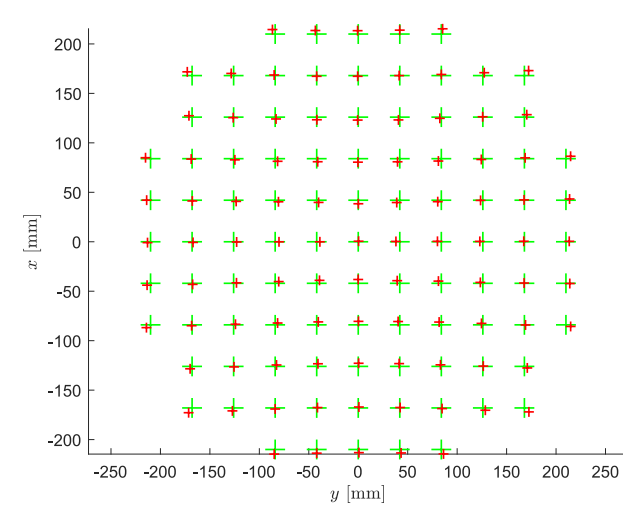

(b) Comparison between the theoretical position of the intersections (large green crosses) and the detected crosses (small red crosses) from figure

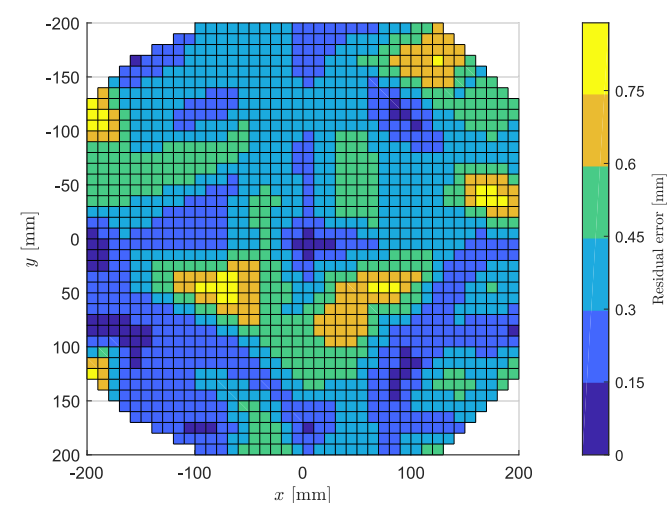

(d) Residual error after calibration of the sphere

Figure 5: Compensation of optical distortion method 


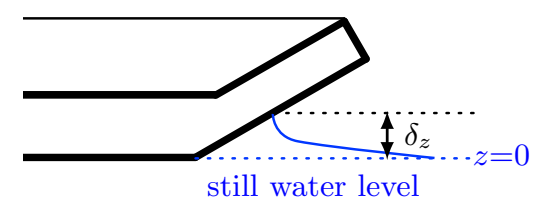

(a) Circular mock-up

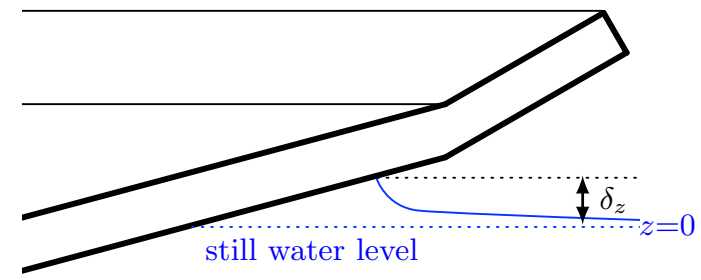

(b) Conical mock-up

Figure 6: Illustration of the meniscus

\subsection{Initial position of the mock-up}

The adjustment of the initial position of the mock-ups in terms of altitude and horizontality is an important feature of the experimental methodology. Different approaches were used for the flat mock-up (circular disc) and the three-dimensional mock-ups (cone and sphere). In the case of the circular disc, the horizontality of the mock-up was first adjusted using an inclinometer $\left(0.05^{\circ}\right.$ resolution) laying on the upper surface of the mock-up. Then the mock-up was lowered vertically step by step until touching the still water. The mock-up became fully wetted within a final step of $0.5 \mathrm{~mm}$. This final position was used as the initial position for all the water exit experiments carried out with the circular disc. Note however that the initial radius of the wetted surface was greater than the flat part of the mock-up because of the presence of a meniscus wetting a portion of the sloped part of the mock-up (see figure 6a).

A different approach was used for the adjustment of the "horizontality" of the threedimensional mock-ups due to the absence of a horizontal plane surface to place the inclinometer. In this case, the mock-up was first partially wetted and then the horizontality was adjusted in order to obtain a symmetric wetted surface (for this purpose, an image of the calibration grid was surperimposed to the image of the camera). Finally, the vertical position of the mock-up was adjusted to obtain the desired value of the wetted surface radius. This position was used as the reference position in the water exit and entry-exit experiments. Note that with this methodology, the size of the wetted surface is influenced by the presence of the meniscus. This means that the boundary of the wetted surface does not correspond to the intersection of the still water surface with the lower surface of the mock-up (see figure $6 \mathrm{~b}$ ).

\subsection{Motion prescribed to the mock-up}

In the water exit experiments, the elevation of the mock-up, $h(t)$, with respect to its initial position follows the following time evolution:

$$
\begin{cases}h(t)=H\left[1+\operatorname{erf}\left(U_{\max }\left(t-t_{0}\right) \sqrt{\pi} / H\right)\right] / 2, & t \leqslant t_{0}, \\ \dot{h}(t)=U_{\max }, & t \geqslant t_{0}\end{cases}
$$

with $\operatorname{erf}(x)=(2 / \sqrt{\pi}) \int_{0}^{x} \exp \left(-\tau^{2}\right) \mathrm{d} \tau$ the error function, $U_{\max }$ the maximum velocity reached during the experiment, $H$ the maximum elevation of the mock-up and $\dot{h}(t)$ the time derivative of $h(t)$. In the water exit experiments, the value of $H$ was fixed to $10 \mathrm{~cm}$, the value of $U_{\max }$ was varied from 0.2 to $0.6 \mathrm{~m} / \mathrm{s}$ and $t_{0}$ was set to $3 \mathrm{~s}$. The time history 


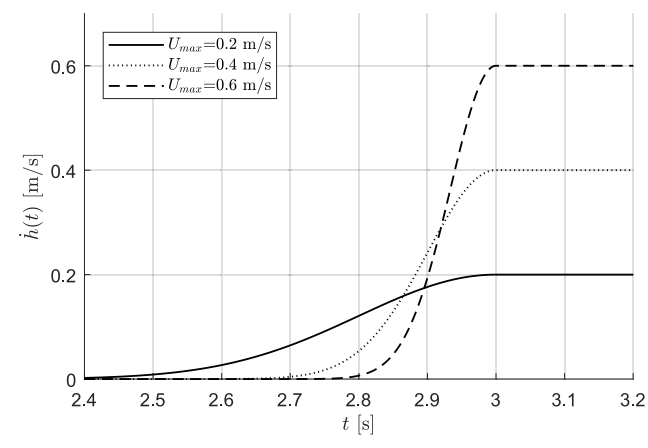

(a) Velocity

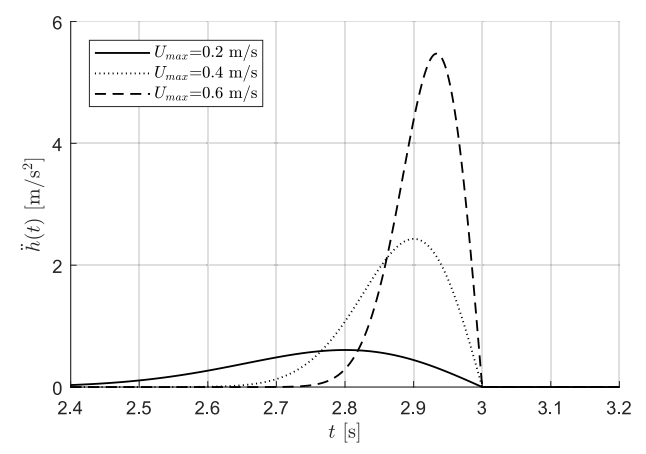

(b) Acceleration

Figure 7: Evolution of the velocity (a) and acceleration (b) as a function of time during the water exit experiments for different values of $U_{\max }$

of the velocity and acceleration for the diffrent values of $U_{\max }$ is illustrated in figure 7 . Formally speaking, $h(t)$ tends to 0 at $t=-\infty$, but in practice $h(0)$ was considered to be sufficiently small to be neglected in the experiments. Note that this motion was inspired by Reis et al. (2010) who originally studied the water exit phenomenon in order to understand the cat lapping phenomenon. Following Reis et al. (2010), the characteristic speed $U_{\max }$ and the initial wetted surface radius $c_{0}$ can be used to calculate the Froude (Fr), Reynolds (Re) and Weber (We) numbers for the water exit experiments: $\mathrm{Fr}=$ $U_{\max } / \sqrt{g c_{0}}, \operatorname{Re}=U_{\max } c_{0} / \nu$ and $\mathrm{We}=\rho c_{0} U_{\max }^{2} / \sigma$, with $g$ the acceleration due to gravity, $\nu$ the liquid kinematic viscosity and $\sigma$ the surface tension coefficient. In the water exit experiments, the value of Fr ranges from $0.13\left(U_{\max }=0.2 \mathrm{~m} / \mathrm{s}, c_{0}=0.25 \mathrm{~m}\right)$ to $0.43\left(U_{\max }=0.6 \mathrm{~m} / \mathrm{s}, c_{0}=0.2 \mathrm{~m}\right), \mathrm{Re} \approx 4.0 \times 10^{4}$ to $1.5 \times 10^{5}$ and $\mathrm{We} \approx 1.1 \times 10^{2}$ to $1.2 \times 10^{3}$.

In the water entry and exit experiments, the elevation of the lowest point of the mockup, $h(t)$, with respect to the free surface altitude follows the following time evolution:

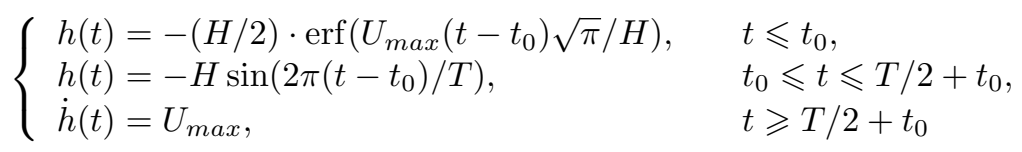

with $U_{\max }$ the maximum velocity and $T=2 \pi H / U_{\max } . H$ is the maximum submergence depth and $t_{0}=3 \mathrm{~s}$. The body enters the water at $t_{0}$ with an initial velocity equal to $-U_{\max }$. The acceleration is maximum at $t=T / 4+t_{0}$ when the body begins to move upward. Note that for $t \leqslant t_{0}$, the motion prescribed to accelerate the mock-up to the desired entry velocity follows the same evolution as for the exit experiments, but in the downward direction. Using the axisymmetric formulation of Wagner's theory, $H$ can be related to $c_{\max }$, the maximum wetted surface radius expected to be reached in an experiment. For the $15^{\circ}$ cone, the Wagner condition (see Korobkin \& Scolan 2006, eq. 48) reads: $H=$ $\pi \tan \left(15^{\circ}\right) c_{\max } / 4$ and for the sphere: $H=\int_{0}^{\pi / 2} \sin \beta\left[R-\sqrt{R^{2}-c_{\max }^{2} \sin ^{2} \beta}\right] \mathrm{d} \beta$, where $R=353.6 \mathrm{~mm}$. The values of the maximum acceleration attained during the different combined entry and exit experiments are reported in table 1. The Froude, Reynolds and Weber numbers can be calculated in a similar way as for the exit experiments, except 


\begin{tabular}{cccc|cccc}
\hline \multicolumn{3}{c}{ Cone } & \multicolumn{4}{c}{ Sphere } \\
\hline$c_{\text {max }}[\mathrm{mm}]$ & $U_{\max }[\mathrm{m} / \mathrm{s}]$ & $\max (\ddot{h})\left[\mathrm{m} / \mathrm{s}^{2}\right]$ & $\operatorname{Fr}$ & $c_{\max }[\mathrm{mm}]$ & $U_{\max }[\mathrm{m} / \mathrm{s}]$ & $\max (\ddot{h})\left[\mathrm{m} / \mathrm{s}^{2}\right]$ & $\mathrm{Fr}$ \\
200 & 0.57 & 7.72 & 0.41 & 200 & 0.56 & 7.74 & 0.40 \\
200 & 0.4 & 3.80 & 0.29 & 200 & 0.4 & 3.95 & 0.29 \\
250 & 0.6 & 6.84 & 0.38 & 250 & 0.6 & 5.41 & 0.38 \\
250 & 0.4 & 3.04 & 0.26 & & & &
\end{tabular}

Table 1: Froude number and maximum acceleration attained during the combined entry and exit experiments for the different values of $c_{\max }$ and $U_{\max }$

that $c_{0}$ should be replaced by $c_{\max }$. The values of Fr corresponding to the experiments are given in table 1 .

\section{Method of experimental analysis}

In this section we describe the method used to analyse the results obtained from the different experiments.

\subsection{Synchronisation of the measurements with the motion generator}

The data acquisition system and the high-speed video camera were triggered by the same signal coming from the motion generator, and so were well synchronised to each other with a maximum time shift of $1 \mathrm{~ms}$ (the sampling frequency and frame rate were both set to $1 \mathrm{kHz}$ ). However, it was not possible to accurately synchronise the motion of the hexapode and the data/video acquisition system during the experiments. In our analysis of the experiments, we compared the theoretical acceleration evolution and the recorded acceleration signal to "re-synchronise" the measurements with the motion. The time shift between the motion and the measured signals was obtained by maximising the cross-correlation between the measured and theoretical acceleration signals. In order to do so, we filtered the high-frequency oscillations of the measured acceleration signals using a Fourier low-pass filter with a cut-off frequency of $30 \mathrm{~Hz}$. An example of the raw and filtered acceleration signals measured during a water exit experiment with $U_{\max }=0.6$ $\mathrm{m} / \mathrm{s}$ is depicted in figure 8. A comparison of the theoretical and measured acceleration signals is presented in figure 9 . The theoretical acceleration signal after applying the same low-pass filter is also depicted in figure 9 to show that the filtering operation does not affect the dynamics of the motion. We can see that the hexapod follows quite well the target motion even if the measured acceleration exhibits a small oscillation which leads to a difference of about $0.28 \mathrm{~m} \mathrm{~s}^{-2}$ in terms of the acceleration peak value. Despite the small oscillations of the acceleration signal, the motion of the hexapod is very repeatable as it can be seen in figure 10 where we compare the filtered acceleration signals recorded during three experiments of exit in air with $U_{\max }=0.6 \mathrm{~m} / \mathrm{s}$. 


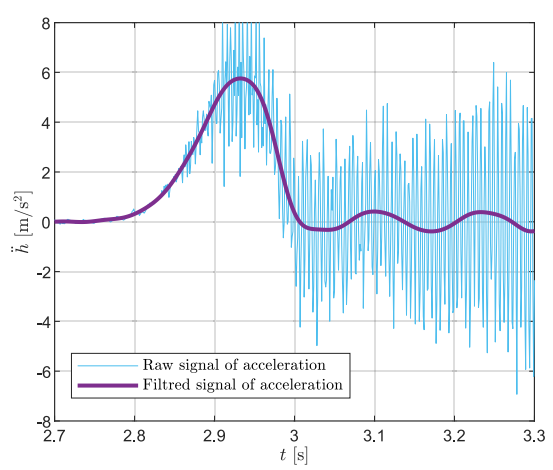

Figure 8: Comparison of the original and filtered measured acceleration signals during a water exit experiment with $U_{\max }=0.6 \mathrm{~m} / \mathrm{s}$

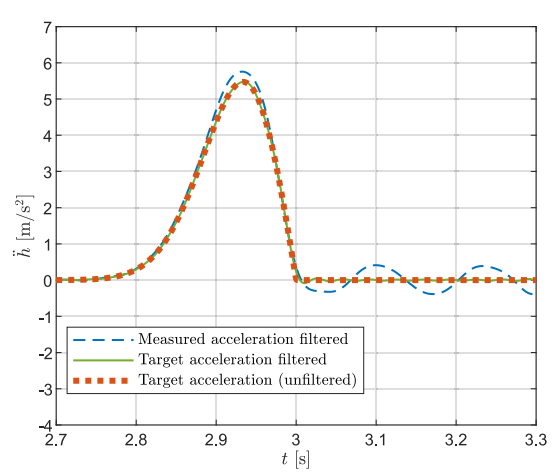

Figure 9: Comparison of the filtered measured and target acceleration (filtered and unfiltered) for water exit with $U_{\max }=0.6 \mathrm{~m} / \mathrm{s}$

\subsection{Tracking of the contact line}

A sequence of four images recorded during the water entry and exit of the cone for different time instants is plotted in figure 11. During the entry stage (figures 11a and $11 \mathrm{~b})$, both the jet front and the contact line are clearly visible. Indeed, they are both illuminated by the LED edge-lighting system. During the exit stage (figures 11c and 11d), the jet disappears but the contact line, which contracts towards the centre, is still clearly visible. Some additionnal concentric luminous rings are nevertheless visible in figures 11c and $11 \mathrm{~d}$. These features are imparted to undesired specular reflections of the very bright outer region of the mock-up on the free-surface, but their presence does not alter the tracking of the contact line which corresponds to the inner luminous ring in the images. In order to measure the evolution of the contact line during the experiments, the contact line geometry is extracted using a contour detection algorithm based on the method of Taubin (1991) and assuming that the shape of the contact line is elliptic. The performance of the contour tracking algorithm is illustrated in figure 12 where the detected contours and the original images (from figure 11) are superimposed. The tracking of the contact line is not problematic, except for a very short duration at the moment of the transition between the entry stage and the exit stage because the contact line is not bright enough. In order to verify that the inner ring corresponds to the contour of the wetted surface (the contact line), the same experiments were conducted with a draughtboard laying at the bottom of the tank (approximately $2 \mathrm{~m}$ below the free surface). With this technique, the region over which the water is in contact with the mock-up corresponds to the region over which the draughtboard appears undistorded (except for the optical distorsion due to the shape of the mock-up), as shown in figure 13. The red dashed line which is superimposed to the images obtained with the draughtboard technique in figure 13 corresponds to the contour extracted from figure 12. One can observe that the region over which the draughtboard remains undistorded is perfectly circumscribed by the contour extracted from figure 12. This comparison demonstrates the accuracy of the LED edge-lighting technique for both water entry and water exit experiments. A more detailed validation of the LED edge-lighting technique through comparison with images obtained with the draughtboard technique for the different mock-ups is presented in appendix A. 


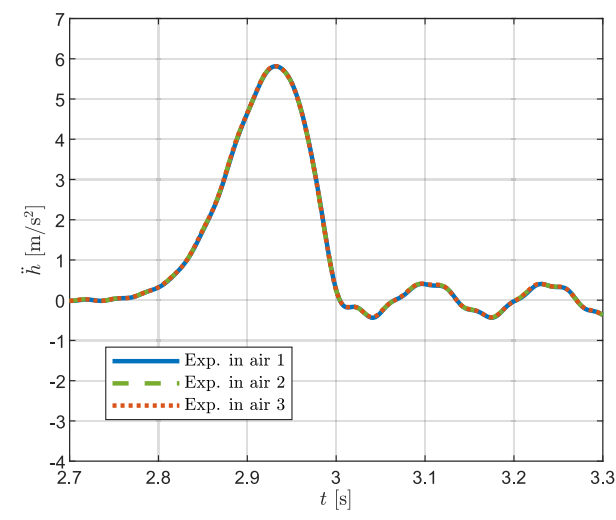

Figure 10: Comparison of the measured acceleration signals for three similar exit experiments in air with $U_{\max }=0.6 \mathrm{~m} / \mathrm{s}$

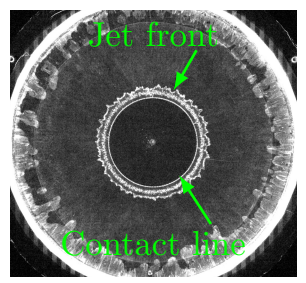

(a) $t=3.036 \mathrm{~s}$

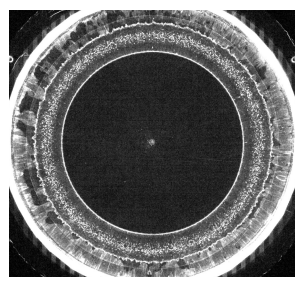

(b) $t=3.073 \mathrm{~s}$

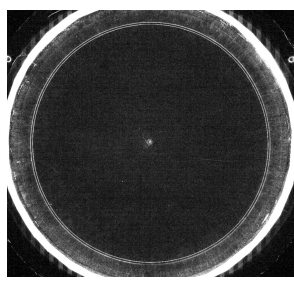

(c) $t=3.166 \mathrm{~s}$

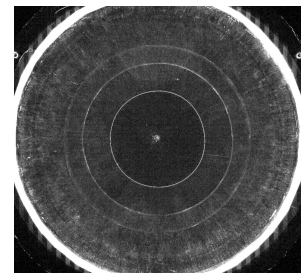

(d) $t=3.318 \mathrm{~s}$

Figure 11: Sequence of images obtained during the water entry $(a, b)$ and exit $(c, d)$ of the cone with $c_{\max }=250 \mathrm{~mm}$ and $U_{\max }=0.6 \mathrm{~m} / \mathrm{s}$

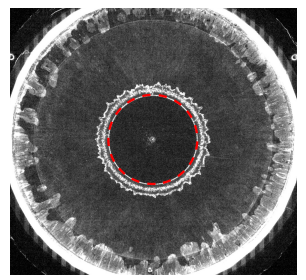

(a) $t=3.036 \mathrm{~s}$

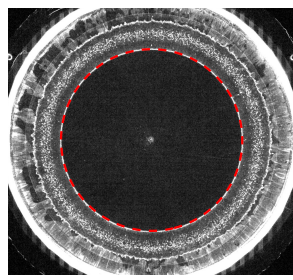

(b) $t=3.073 \mathrm{~s}$

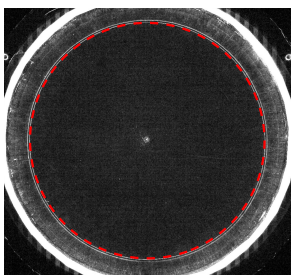

(c) $t=3.166 \mathrm{~s}$

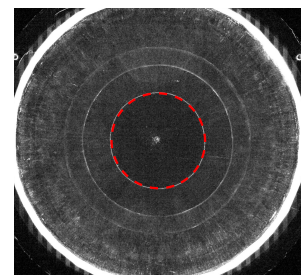

(d) $t=3.318 \mathrm{~s}$

Figure 12: Tracking of the contact line during the water entry $(\mathrm{a}, \mathrm{b})$ and exit $(\mathrm{c}, \mathrm{d})$ of the cone

\subsection{Hydrodynamic force measurement}

In our analysis procedure, the force signals are first low-pass filtered with the same filter as the acceleration signal. It was shown previously that the filtering operation did not affect the acceleration signal (see figure 9). Given that the hydrodynamic force during water exit is principally due to an "added mass-like" effect (see Tassin et al. 2012; Korobkin 2013), we can anticipate that the filtering will not affect the force signals (except for the removal of the high-frequency oscillations). The force measured by the sensors is a combination of the action of the water on the mock-up and the inertia force due to the mass of the mock-up and of the mechanical parts connecting the mock-up to the force sensors. In order to substract the force component due to inertia from the total 


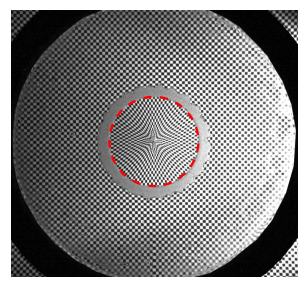

(a) $t=3.036 \mathrm{~s}$

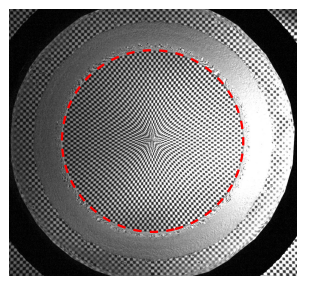

(b) $t=3.073 \mathrm{~s}$

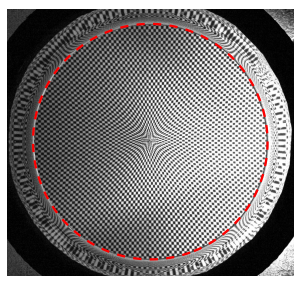

(c) $t=3.166 \mathrm{~s}$

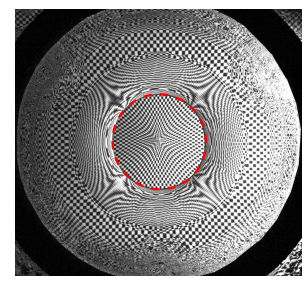

(d) $t=3.318 \mathrm{~s}$

Figure 13: Comparison between the contact line obtained with the LED edge-lighting technique (red dashed line) and the images obtained with the draughtboard during the water entry and exit of the cone

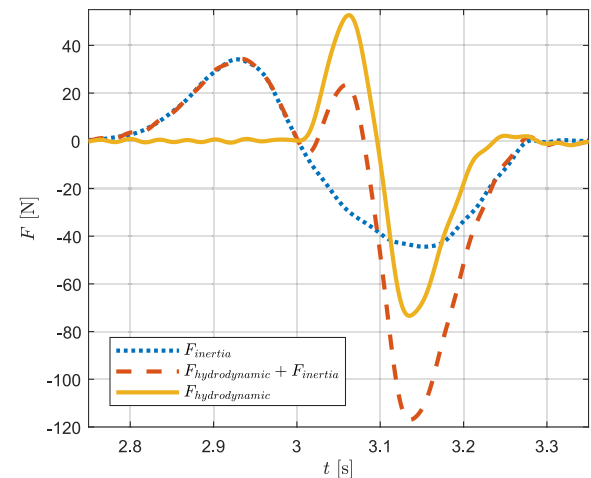

(a) Water entry and exit of a cone with $U_{\max }=0.6 \mathrm{~m} / \mathrm{s}$ and $c_{\max }=250 \mathrm{~mm}$

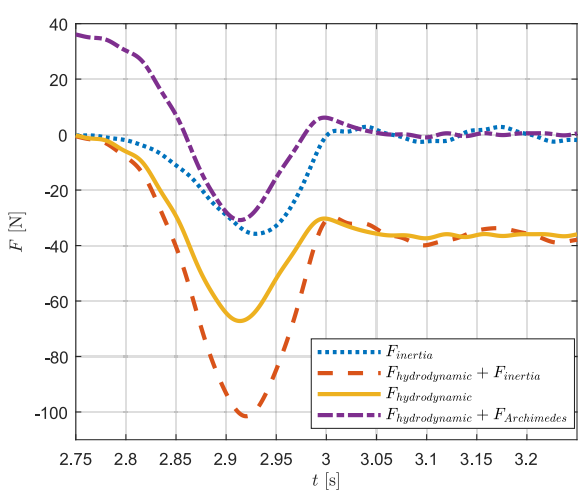

(b) Water exit of a cone with $U_{\max }=0.6 \mathrm{~m} / \mathrm{s}$ and $c_{0}=250 \mathrm{~mm}$

Figure 14: Contribution of the different force components to the total force

measured force, the inertia component was measured by running experiments in air with a motion identical to the experiments with water. Each experiment in air and water was repeated three times with the same prescribed motion. The measured total force and the contribution of the different force components during an experiment of water entry and exit with the cone are plotted in figure 14a. One can appreciate the relative importance of the inertia component $\left(F_{\text {inertia }}\right)$ to the total force.

The use of piezoelectric force sensors, even with a slow-drift charge amplifier like the one we used, requires a reset of the level of reference of the force sensors at the beginning of the experiment. As a consequence, the measured hydrodynamic force is equal to zero at the beginning of the pure exit experiment, even for the three-dimensional mock-ups, despite the action of Archimedes' force. In the results presented in the following sections, the reset of the force sensors is corrected by substracting the final asymptotic value of the hydrodynamic force (when the mock-up is fully unwetted and the velocity is null). The contribution of the different force components during the water exit of the cone are shown in figure 14b below.

\subsection{Correction of the reference elevation in the water entry and exit experiments}

The presence of the meniscus induces an uncertainty of $\delta_{z}$ (corresponding to the meniscus height) on the initial position of the three-dimensional mock-ups (with respect to the still water level) . However, the height of the meniscus can be estimated from 


$\begin{array}{lcccc}c_{0}[\mathrm{~mm}] & U_{\max }[\mathrm{m} / \mathrm{s}] & \delta_{t}[\mathrm{~s}] & \left|h\left(t_{0}+\delta_{t}\right)\right|[\mathrm{mm}] & \delta_{z}^{*}[\mathrm{~mm}] \\ 250 & 0.6 & 0.005 & 3.0 & 3.6 \\ 200 & 0.57 & 0.0048 & 2.8 & 3.8\end{array}$

Table 2: Estimation of the meniscus height $\delta_{z}$ for the cone $: \delta_{z}^{*}$ is the estimate of $\delta_{z}$ based on the measured value of Archimedes' force and $\left|h\left(t_{0}+\delta_{t}\right)\right|$ is the estimate of $\delta_{z}$ based on the measured value of $\delta_{t}$

$\begin{array}{lcccc}c_{0}[\mathrm{~mm}] & U_{\max }[\mathrm{m} / \mathrm{s}] & \delta_{t}[\mathrm{~s}] & \left|h\left(t_{0}+\delta_{t}\right)\right|[\mathrm{mm}] & \delta_{z}^{*}[\mathrm{~mm}] \\ 250 & 0.6 & 0.008 & 4.8 & 4.3 \\ 200 & 0.56 & 0.007 & 3.9 & 4.2\end{array}$

Table 3: Estimation of the meniscus height $\delta_{z}$ for the sphere $: \delta_{z}^{*}$ is the estimate of $\delta_{z}$ based on the measured value of Archimedes' force and $\left|h\left(t_{0}+\delta_{t}\right)\right|$ is the estimate of $\delta_{z}$ based on the measured value of $\delta_{t}$

the experiments in two ways. The first way is to find a value of $\delta_{z}$ such that the theoretical buoyancy force is equal to the measured force at the beginning of the water exit experiment. The second way is to detect from the high-speed videos the time instant when the mock-up touches the water $\left(t=t_{0}+\delta_{t}\right)$ and to compute the theoretical elevation of the mock-up at this time instant $\left(h\left(t_{0}+\delta_{t}\right)\right)$. The values obtained for $\delta_{z}$ with the two approaches for the cone and the sphere are given in tables 2 and 3, respectively. One can see that the maximum difference between the two estimates is about $1 \mathrm{~mm}$. Note that these values will be used later in order to make comparisons with the theoretical results.

\section{Experimental results}

In this section we present the experimental results in terms of the evolution of the radius of the wetted surface and of the hydrodynamic force measured during the experiments of pure water exit (section 4.1) and combined water entry and exit (sections 4.2 and 4.3).

\subsection{Evolution of the wetted surface and of the hydrodynamic force during the water exit experiments}

The evolution of the radius of the wetted surface during the water exit of the circular disc is depicted in figure 15 for different values of the maximum exit velocity. The corresponding force evolutions are presented in figure 16. One can observe in figure 15 that the evolution of the wetted surface radius depends on the exit velocity. For a given disc elevation, the radius is smaller when the velocity is decreased. This phenomenon is due to the action of the gravity. As the maximum exit velocity decreases, the peak (absolute) value of the hydrodynamic force decreases and occurs earlier.

The results obtained with the cone are depicted in figures 17 and 18. Similarly to the circular disc, the reduction of the wetted surface radius with the elevation is more pronounced as the velocity decreases. In figure 17, the evolution of the radius of the wetted surface obtained with a von Karman approach $\dagger$ is also plotted. The difference

$\dagger$ Within the von Karman approach, the contact line position is determined as the intersection 


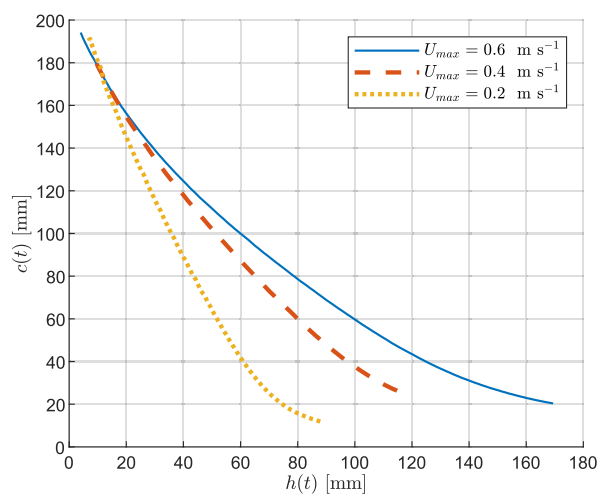

Figure 15: Evolution of the radius of the wetted surface as a function of the elevation during the water exit of the circular disc for different values of $U_{\max }$

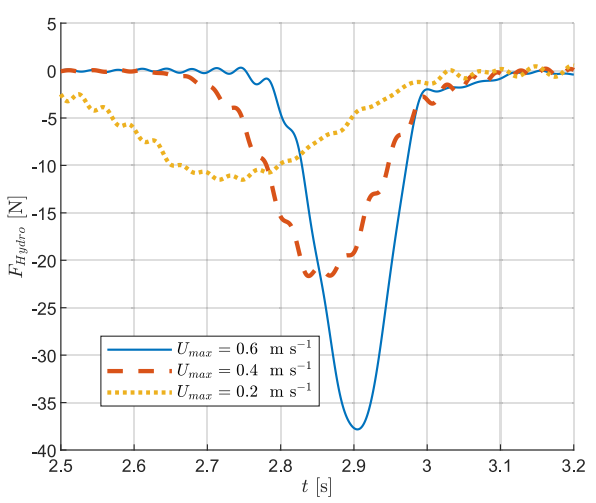

Figure 16: Evolution of the hydrodynamic force as a function of time during the water exit of the circular disc for different values of $U_{\max }$

between the experimental results and the results obtained with the von Karman approach illustrates the pile-up of the free surface induced by the water exit. The hydrodynamic force evolutions measured during the water exit of the cone differ from that measured during the water exit of the circular disc due to the static buoyancy of the cone. The buoyancy force mitigates the suction force in the case of the cone. For the lowest velocity $\left(U_{\max }=0.2 \mathrm{~m} / \mathrm{s}\right)$, the hydrodynamic force remains positive during the entire duration of the exit experiment. In agreement with what can be expected from a theoretical model of exit based on an added mass approach, the hydrodynamic force measured during the water exit of the disc and the cone becomes very low when the acceleration vanishes (at $t=3 \mathrm{~s}$ ). However, it is not equal to zero. Indeed, a drag force is observed for $t>3 \mathrm{~s}$. This force decreases gradually with time, most likely because of the decrease of the size of the wetted surface.

The results obtained during the water exit of the sphere are presented in figures 19 and 20. In this case, the velocity has a much smaller influence on the evolution of the radius of the wetted surface. One may expect that the effect of the gravity would be more pronounced in the case of the sphere due to the larger volume displaced by the mock-up, but the difference between the experimental results and the results obtained with the von Karman approach (also plotted in figure 19) demonstrates that the phenomenon is not quasi-static. Also note that the hydrodynamic force remains positive during almost the entire duration of the experiments, even for the highest maximum velocity $(0.6 \mathrm{~m} / \mathrm{s})$, due to the importance of the buoyancy force.

\subsection{Water entry and exit of the cone}

The evolutions of the radius of the wetted surface during the combined water entry and exit of the cone are presented in figures 21 and 22 for $c_{\max }=200 \mathrm{~mm}$ and $c_{\max }=250$ $\mathrm{mm}$, respectively. The maximum radius, $c_{\max }$, corresponds to the theoretical value of the radius of the wetted surface obtained with the Wagner model when the penetration depth is maximum (at $t=t_{0}+T / 4$ ). Note that the maximum value of $c_{\max }$ is not attained during

between the body surface and the reference horizontal plane $z=\delta_{z}$ (represented by a black dotted line in figure $6 \mathrm{~b}$ ) 


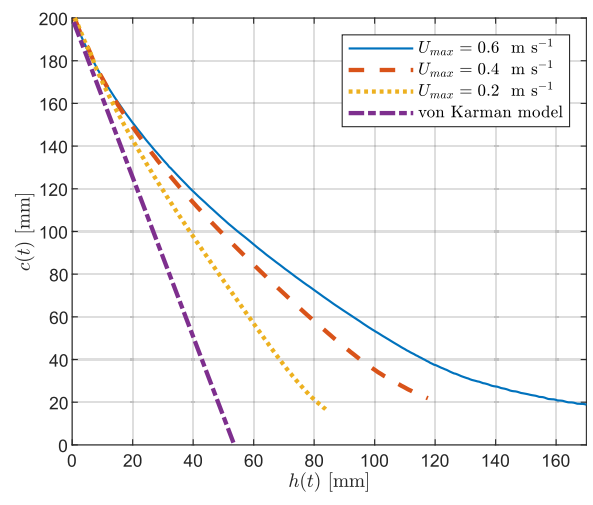

Figure 17: Evolution of the radius of the wetted surface as a function of the elevation during the water exit of the cone for different values of $U_{\max }$

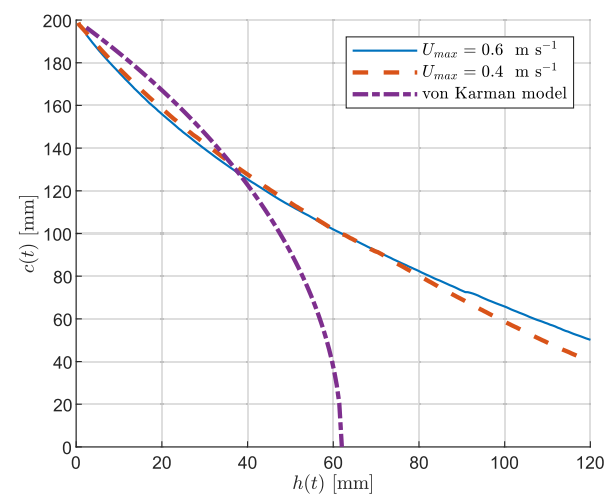

Figure 19: Evolution of the radius of the wetted surface as a function of the elevation during the water exit of the sphere for different values of $U_{\max }$

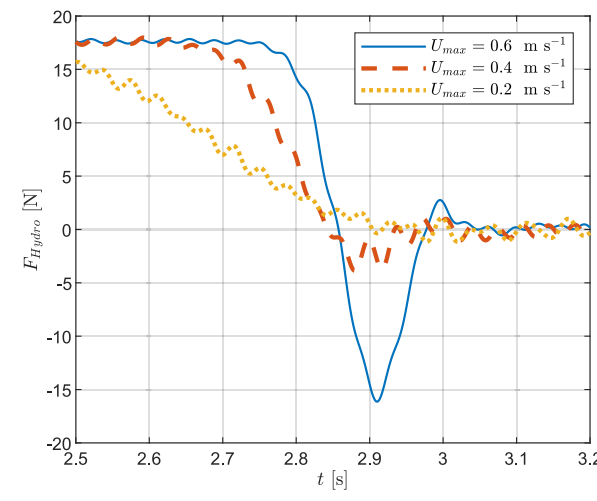

Figure 18: Evolution of the hydrodynamic force as a function of time during the water exit of the cone for different values of $U_{\max }$

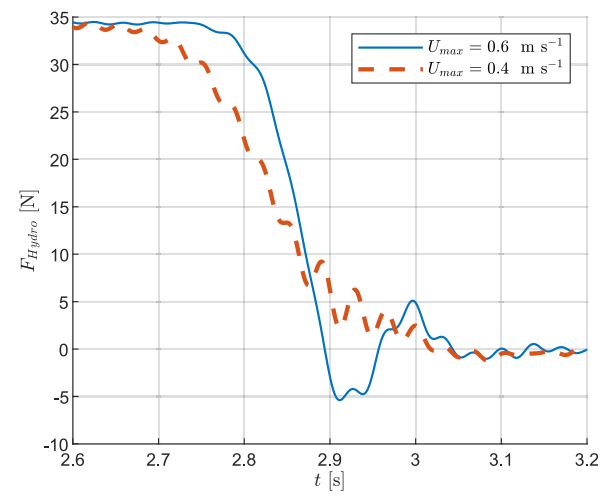

Figure 20: Evolution of the hydrodynamic force as a function of time during the water exit of the sphere for different values of $U_{\max }$

the experiments. We will see later that this can be explained by a value of $\delta_{z} \approx 3 \mathrm{~mm}$. The value of the maximum velocity $\left(U_{\max }\right)$ has little effect on the maximum value of the radius of the wetted surface. This indicates that gravity does not affect the wetted surface evolution during the entry stage (for the considered entry velocities). The evolution of the hydrodynamic force for $c_{\max }=200 \mathrm{~mm}$ and for $c_{\max }=250 \mathrm{~mm}$ is shown in figures 23 and 24, respectively. According to the image recordings, the cone starts touching the water at $t=t_{0}+\delta_{t} \approx 3.005 \mathrm{~s}$ (see table 2 ). After that, the load begins to increase and reaches rapidly a maximum. Then, the load decreases and becomes negative (corresponding to suction forces). It should be noted that at the time when the force is equal to zero, the mock-up velocity is still downward. The duration of the stage during which the force is negative is longer than the one during which it is positive. Also, the maximum force, in terms of magnitude, corresponds to a suction load (directed downward) and is reached at the transition between the entry and exit stages. This observation is in line with the 


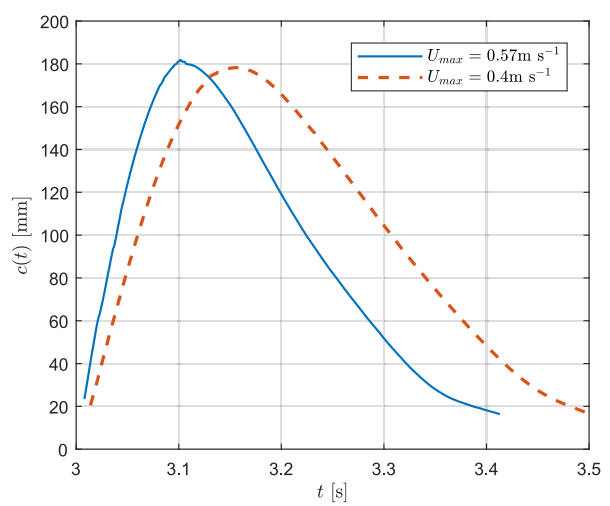

Figure 21: Evolution of the radius of the contact line as a function of time during the water entry and exit of the cone for $c_{\max }=200 \mathrm{~mm}$

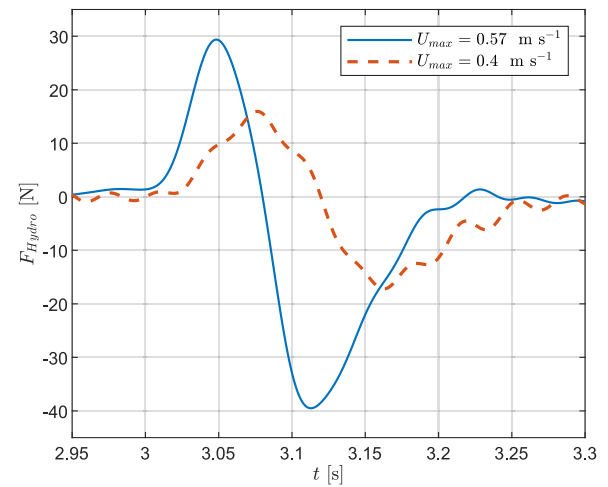

Figure 23: Evolution of the hydrodynamic force as a function of time during the water entry and exit of the cone for $c_{\max }=200 \mathrm{~mm}$

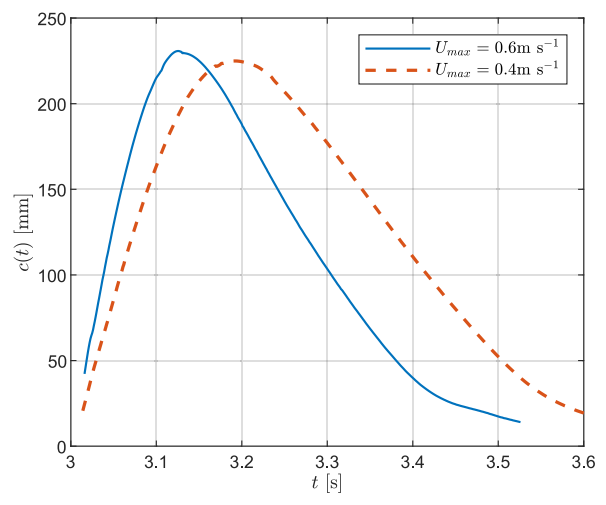

Figure 22: Evolution of the radius of the contact line as a function of time during the water entry and exit of the cone for $c_{\max }=250 \mathrm{~mm}$

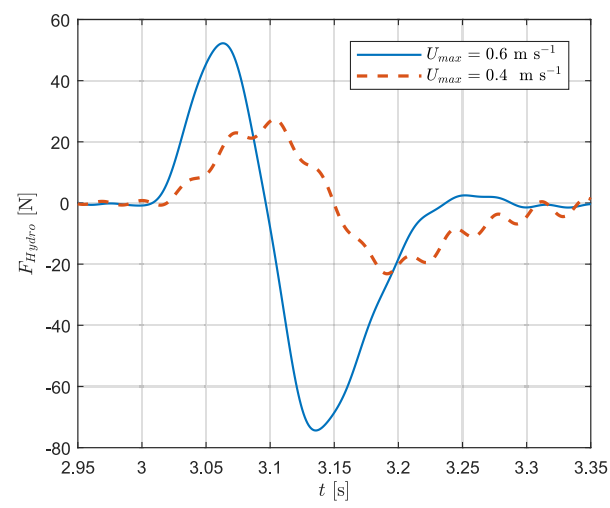

Figure 24: Evolution of the hydrodynamic force as a function of time during the water entry and exit of the cone for $c_{\max }=250 \mathrm{~mm}$

experimental observations and theoretical predictions of Baarholm \& Faltinsen (2004) and Tassin et al. (2013). Note that the force results measured during the experiments with $U_{\max }=0.4 \mathrm{~m} / \mathrm{s}$ exhibit more oscillations than the results obtained with $U_{\max }=0.57$ $\mathrm{m} / \mathrm{s}$.

\subsection{Water entry and exit of the sphere}

The evolution of the hydrodynamic force during the combined water entry and exit of the sphere is presented in figures 25 and 26 for $c_{\max }=200 \mathrm{~mm}$ and $c_{\max }=250 \mathrm{~mm}$, respectively. Note that the cut-off fequency was set to $25 \mathrm{~Hz}$ instead of $30 \mathrm{~Hz}$ for the results presented in figure 26 in order to reduce the oscillations of the force signal due to the vibrations of the set-up. One can observe that, in contrast to the water entry and exit of the cone, the maximum magnitude of the force is more important during the entry stage than during the exit stage. Due to a weak contrast in the images, it has not 


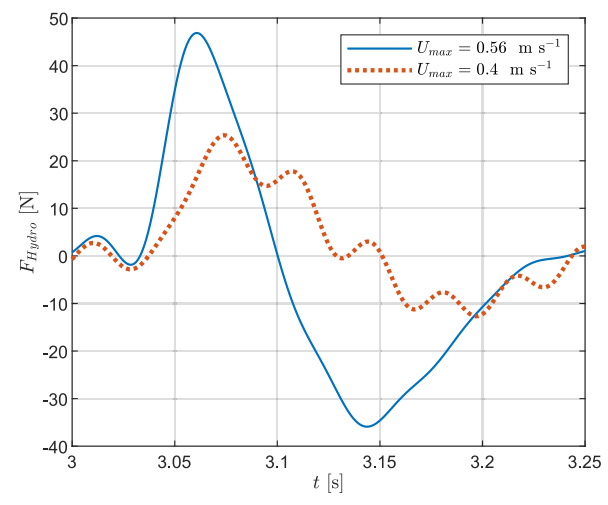

Figure 25: Evolution of the hydrodynamic force as a function of time during the water entry and exit of the sphere for two different velocities and a theoretical $c_{\max }=200 \mathrm{~mm}$

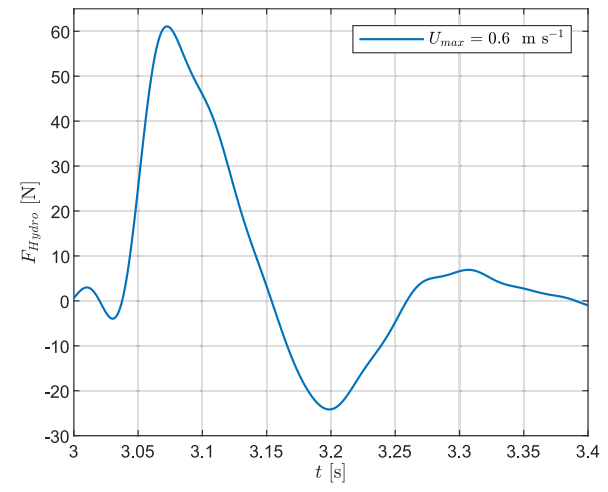

Figure 26: Evolution of the hydrodynamic force as a function of time during the water entry and exit of the sphere for a theoretical $c_{\max }=250 \mathrm{~mm}$

been possible to use the automatic contact line tracking algorithm for the entry and exit experiments carried out with the sphere.

\section{Investigation of different parameters affecting the water exit phenomenon and comparisons with theoretical results}

In this section we investigate the effect of different parameters that influence the water exit phenomenon. We study the effect of the scale, the effect of the shape of the mock-up and the impact of the entry stage on the results of the exit stage. We also compare the measurements with theoretical results.

\subsection{Effect of the scale (experiments in Froude similarity)}

At present, there are still some open questions regarding the mechanisms governing the wetted surface dynamics in water exit problems. In particular, the question of the effect of surface tension and viscosity at the early stage of a water exit event was raised by Korobkin (2013). In order to assess the importance of these effects in our experiments, we conducted experiments in Froude similarity with the cone for different values of the initial wetted surface, hence varying the scale of the experiments (so varying the Reynolds and Weber numbers) while keeping the Froude number constant. The initial value of the radius of the wetted surface was varied from $c_{0}=250 \mathrm{~mm}$ to $c_{0}=100 \mathrm{~mm}$. The scaling was based on a reference maximum velocity $U_{\max }=0.6 \mathrm{~m} / \mathrm{s}$, a maximum elevation height $H=0.1 \mathrm{~m}$ and $c_{0}=0.25 \mathrm{~m}$. Assuming that $\alpha=c_{0} / 0.25$ (with the value of $c_{0}$ in meters) represents the scale of the experiment, the function $h(t)$ defining the motion of the cone for the different values of $c_{0}$ is given by equation 2.2 , with $H=\alpha \times 0.1 \mathrm{~m}$, $U_{\max }=\sqrt{\alpha} \times 0.6 \mathrm{~m} / \mathrm{s}$ and $t_{0}=\sqrt{\alpha} \times 3 \mathrm{~s}$. The results obtained for the diffrent values of $c_{0}$ are compared in figure 27 in terms of the nondimensional radius of the wetted surface as a function of the nondimensional elevation. One can see that the results obtained at different scales are in very close agreement. Note however that the results obtained with $c_{0}=100 \mathrm{~mm}$ slightly deviate from the others at the end of the exit, but this difference remains within the accuracy margin of the experimental approach because of 


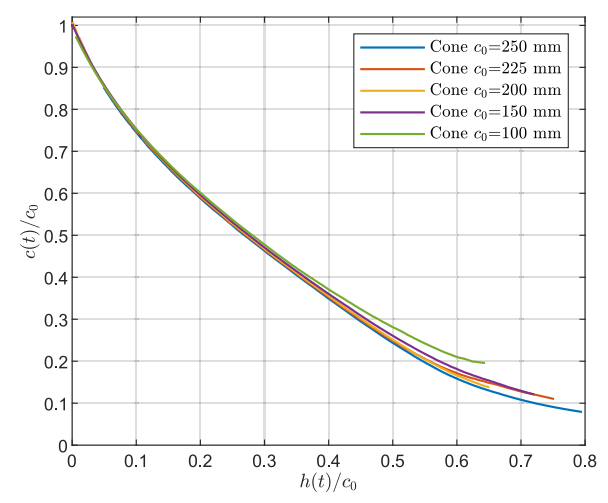

Figure 27: Evolution of the nondimensional radius of the wetted area $c(t) / c_{0}$ as a function of the nondimensional elevation during the water exit of the cone for different values of $c_{0}$ in Froude similarity

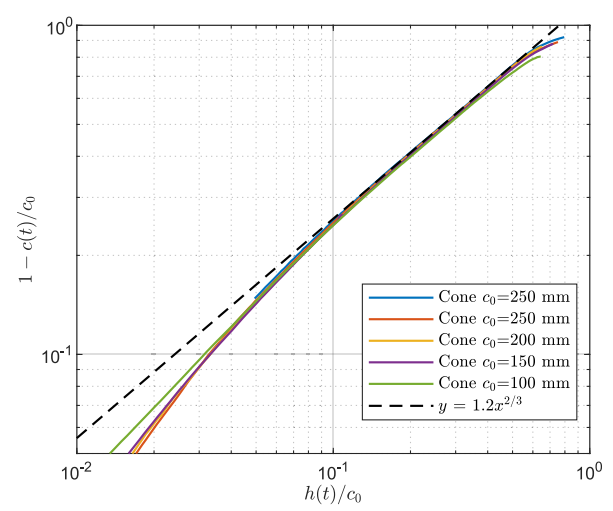

Figure 28: Evolution of $1-c(t) / c_{0}$ as a function of the non-dimensional elevation during the water exit of the cone for different values of $c_{0}$ in Froude similarity with a log-log scale

the imperfections at the center of the conical mock-up (see figure $5 \mathrm{c}$ ). These experiments show that the influence of surface tension and viscosity is marginal at the largest scales and that the dynamics of the wetted surface is mainly controlled by inertia and gravity effects. Following the theoretical work of Korobkin et al. (2017b) who showed that the quantity $1-c(t) / c_{0}$ was proportional to $\left[h(t) / c_{0}\right]^{2 / 3}$ during the water exit of a flat plate at constant acceleration, the experimental results obtained in Froude similarity are depicted in figure 28 in terms of the quantity $1-c(t) / c_{0}$. One can see that the results compare very well with the power-law predicted by Korobkin et al. (2017b) (but with a different coefficient) for $h(t) / c_{0}$ ranging from 0.1 to 0.7 . This agreement is somewhat surprising given the influence of gravity in our experiments (see figure 17) and the rather different motion imposed to the cone.

\subsection{Effect of the exit velocity}

In this section, the effect of the exit velocity $\left(U_{\max }\right)$ on the evolution of the contact surface during the water exit experiments is analysed by plotting the quantity $1-c(t) / c_{0}$ as a function of the non-dimensional elevation $h(t) / c_{0}$. The results obtained for the disc, the cone and the sphere are plotted in figures 29, 30 and 31, respectively. The experimental results of Vega-Martínez et al. (2019) corresponding to the water exit of an elastic circular disc are also shown for comparison in figure 29, but note that their experimental results are limited to $c(t) / c_{0}>0.9$. Two important conclusions can be drawn from these observations. First, the time history of the velocity (for $U_{\max }$ sufficiently large), the elasticity and the shape of the body do not seem to affect significantly the evolution of the radius of the wetted surface with the body elevation. Second, the theoretical prediction of Korobkin et al. (2017b), which is based on a self-similar smalltime expansion, seems to be valid for values of $h(t) / c_{0}$ as large as 0.7 . However, as the value of $U_{\max }$ decreases, the power coefficient evolves. This effect, clearly visible for $U_{\max }=0.2 \mathrm{~m} / \mathrm{s}$ in figures 29 and 30 , is due to the influence of gravity. 


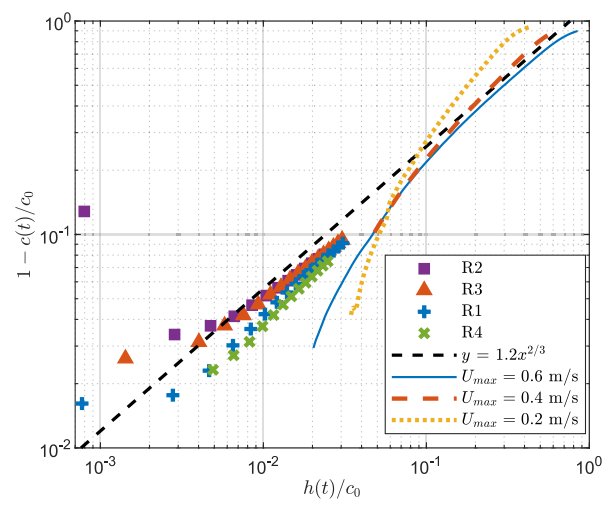

Figure 29: Evolution of $\left[1-c(t) / c_{0}\right]$ as a function of the non-dimensional elevation during the water exit of the disc for different values of $U_{\max }$. The plots denoted by $R 1, R 2, R 3$ and $R 4$ correspond to four experimental results obtained by Vega-Martínez et al. (2019) with a $10.8 \mathrm{~cm}$ radius circular disc

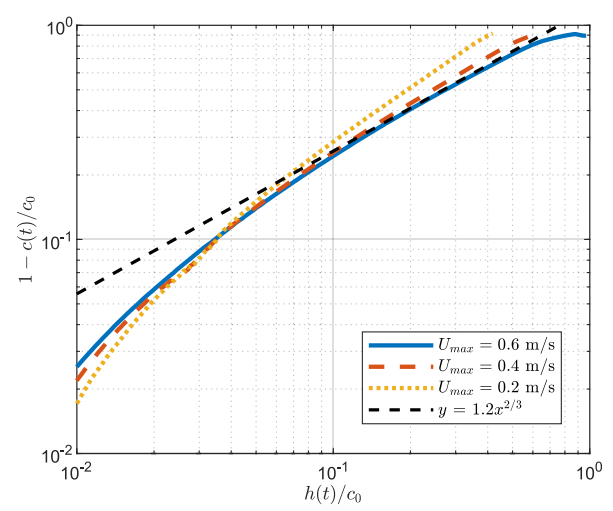

Figure 30: Evolution of $\left[1-c(t) / c_{0}\right]$ as a function of the non-dimensional elevation during the water exit of the cone for different values of $U_{\max }$

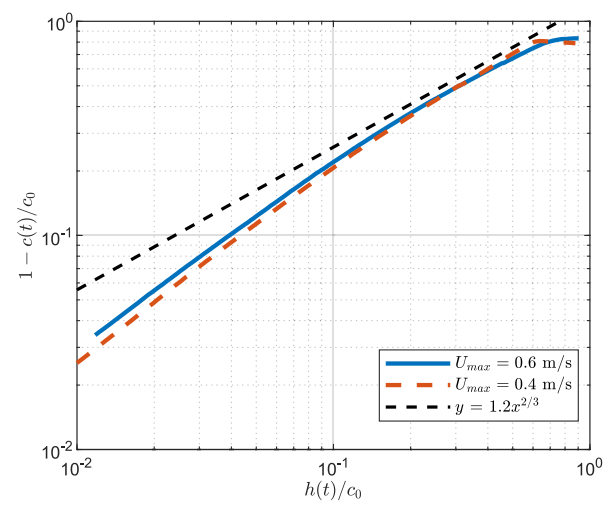

Figure 31: Evolution of $\left[1-c(t) / c_{0}\right]$ as a function of the non-dimensional elevation during the water exit of the sphere for different values of $U_{\max }$

\subsection{Effect of the body shape}

In the linearized water exit model proposed by Korobkin (2013), and further developped in Korobkin et al. $(2017 a)$, the body shape has no effect on the evolution of the wetted surface, provided that the size of the initial wetted surface and the body motion are similar. In order to confirm experimentally this assumption, we compare the results obained with the different body shapes (circular disc, cone, sphere), but with a similar value of $c_{0}$ and a similar motion. Figure 32 shows the evolution of the radius of the wetted surface as a function of the elevation $h(t)$ measured during the water exit of the three mock-ups for $c_{0}=200 \mathrm{~mm}$. One can see that the evolution of the radius of the wetted surface for the cone and the disc are very close, except that the dewetting of the disc is 


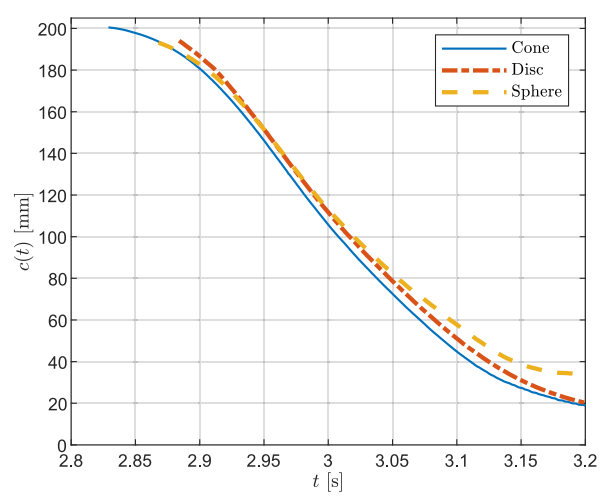

Figure 32: Effect of the body shape on the evolution of the radius of the wetted area $c(t)$ during water exit experiments $\left(U_{\max }=0.6 \mathrm{~m} . \mathrm{s}^{-1}\right.$ and $\left.c_{0}=200 \mathrm{~mm}\right)$

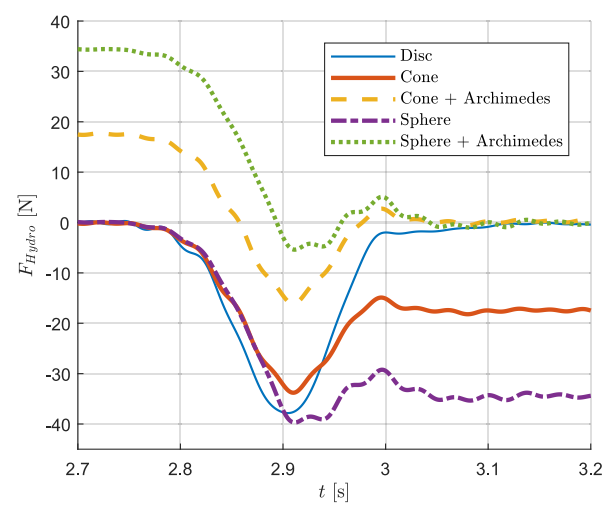

Figure 33: Effect of the body shape on the evolution of the hydrodynamic force $F_{\text {hydro }}$ during water exit experiments $\left(U_{\max }=0.6 \mathrm{~m} \cdot \mathrm{s}^{-1}\right.$ and $\left.c_{0}=200 \mathrm{~mm}\right)$

delayed by a small time shift. This time shift may come from the fact that the initial radius of the wetted surface is slightly larger than $200 \mathrm{~mm}$ because of the meniscus. The evolution of the sphere seems to follow the evolution of the cone at the beginning of the experiments and then approaches the evolution of the disc. This comparison shows that the body shape has a marginal effect on the evolution of the wetted surface during water exit. This observation is consistent with the water exit model of Korobkin (2013) which is insensitive to the shape of the body exiting water (except for the size of the initial wetted surface). The hydrodynamic force measured during the experiments with the different body shapes is shown in figure 33. The total hydrodynamic force measured with the different mock-ups is largely affected by the importance of Archimedes' force. The results obtained after substracting the initial value of the force are also depicted in figure 33 in order to compare the "purely dynamic" contribution to the total hydrodynamic force in the different cases. This comparison shows that the dynamic contribution is rather close for the three shapes at the beginning of the exit (until $t \approx 2,9 \mathrm{~s}$ ).

\subsection{Effect of the entry stage on the results of the exit stage}

In the case of a combined water entry and exit event, the fluid is set in motion and the water free surface is deformed during the entry stage, and one may ask if the exit stage (when it takes place after an entry stage) may differ from a pure water exit experiment. In order to assess the effect of the entry stage on the exit stage, we compare the evolution of the wetted surface during an experiment of pure water exit (for $U_{\max }=0.6 \mathrm{~m} / \mathrm{s}$ ) and during the exit stage of an experiment of combined water entry and exit (for $U_{\max }=$ $0.57 \mathrm{~m} / \mathrm{s}$ ) using the conical mock-up. We can see in figure 34 that, even if the dynamics of the mock-up is not exactly the same in both cases, the evolution of the radius of the wetted surface in the pure-exit experiment is comparable to the one observed during the exit stage of a combined water entry and exit experiment. Note that the radius of the wetted surface measured during the combined water entry and exit experiments is nondimensionalised with respect to the maximum value measured during the experiment. The same results are plotted in logarithmic scale in figure 35. One can observe the very close agreement between the results measured during the exit stage of the combined water entry and exit experiment and the power-law predicted by the theory of Korobkin 


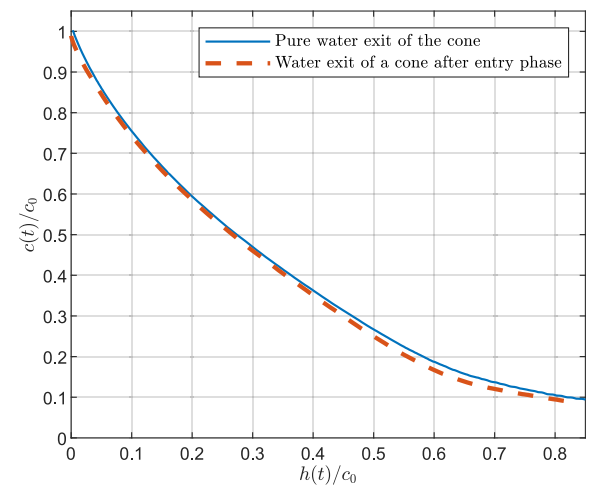

Figure 34: Evolution of the nondimensional radius of the wetted surface $c(t) / c_{0}$ as a function of the non-dimensional elevation $h(t) / c_{0}$ with $c_{0}=200 \mathrm{~mm}$

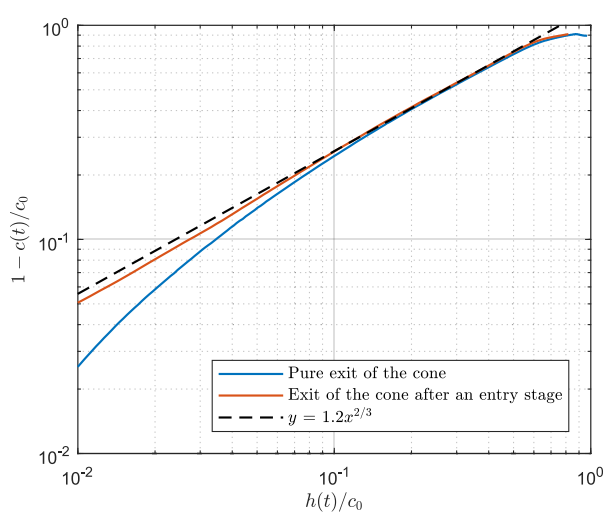

Figure 35: Evolution of the nondimensional radius of the wetted surface $c(t) / c_{0}$ as a function of the non-dimensional elevation $h(t) / c_{0}$ with $c_{0}=200 \mathrm{~mm}$ and in log-log scale

et al. $(2017 b)$. These observations lead us to the important conclusion that a similar water exit model can be used to model both a pure water exit event and the exit stage of a combined water entry and exit event (e.g. in a $2 \mathrm{D}+\mathrm{t}$ approach such as the one used in Bensch et al. (2001), see also Tassin et al. (2013) and Korobkin et al. (2017a)).

\subsection{Comparison with theoretical results}

In this section we compare the experimental results with theoretical results obtained with different analytical models of water entry and exit. In figure 36, the evolution of the wetted surface measured during the water exit experiments with the circular disc is compared to the analytical model of Korobkin et al. (2017a), which is an extension of the model initially proposed in Korobkin (2013) to an arbitrary time evolution of the acceleration of the body. In the axisymmetric version of this model, the hydrodynamic force, $F$, is given by the added mass and the acceleration of the body:

$$
F(t)=-\frac{4}{3} \rho c^{3}(t) \ddot{h}(t),
$$

and the evolution of the radius of the contact surface, $c(t)$, is governed by the following differential equation:

$$
\frac{\mathrm{d} c}{\mathrm{~d} t}=-\frac{2}{\pi} \gamma c(t) \int_{t_{1}}^{t} \frac{\ddot{h}(\tau) \mathrm{d} \tau}{\sqrt{c^{2}(\tau)-c^{2}(t)}},
$$

where $t_{1}=-\infty$ for the pure water exit experiments and $t_{1}=t_{0}+T / 4$ in the combined water entry and exit experiments. We follow the methodology proposed in Korobkin et al. $(2017 a)$ to solve equation 5.2 , but once the solution is well initialised, we solve directly equation 5.2 with a standard first order explicit Euler integration scheme because the methodology proposed in Korobkin et al. (2017a) is no longer valid when the body acceleration $\ddot{h}(t)$ is equal to 0 . It is important to have in mind that gravity effects are neglected in this model, but quantitative comparisons with the experimental results can be made during the early stage of the exit. Indeed, the very close agreement between the experimental results obtained for $U_{\max }=0.6 \mathrm{~m} / \mathrm{s}$ and $U_{\max }=0.4 \mathrm{~m} / \mathrm{s}$ for $h(t)<30 \mathrm{~mm}$ 
indicates that gravity has very little effect on the evolution of the wetted surface during this stage. The theoretical results obtained with $\gamma=1$ are closer to the experimental results obtained for $U_{\max }=0.6 \mathrm{~m} / \mathrm{s}$ and $U_{\max }=0.4 \mathrm{~m} / \mathrm{s}$ at the beginning of the exit, but the theoretical model overestimates the width of the wetted surface as the elevation increases. This divergence between the theoretical and the experimental results is due to the gravity effect which increases as $h(t)$ increases. With $\gamma=2$, the theoretical model seems to underestimate significantly the width of the wetted surface. From figure 36, it seems that there is a slight shift between the experimental and theoretical (for $\gamma=1$ ) wetted surface radius evolutions. The reason for that is probably due to the fact that the initial value of the wetted surface in the experiments is slightly larger than the disc radius, because of the presence of a meniscus (see figure 6). The same problem does not occur during the water exit of the cone because the initial altitude of the mock-up was adjusted such as to have the desired value of $c_{0}$ (see section 2.3). Figure 37 presents a comparison between the predictions of the linearized water exit model of Korobkin et al. $(2017 a)$ and the experimental wetted surface evolutions obtained with the cone for different exit velocities. For low values of the elevation $h(t)$, the evolution of the wetted surface radius is independent of the exit velocity. This corresponds to the stage where gravity effects are negligible. During this stage, the results obtained from the model of Korobkin et al. $(2017 a)$ with $\gamma=1$ are in excellent agreement with the experimental results. In figure 38 , we compare the hydrodynamic force measured during the water exit of the circular disc with the hydrodynamic force predicted by the added mass approach (equation 5.1) and using the value of $c(t)$ measured during the experiments. Note that we were not able to measure the width of the wetted surface at the very beginning of the experiments, so we used the first measured value of $c(t)$ to calculate the hydrodynamic force at the beginning of the exit. Figure 38 shows that the added mass approach seems to provide a good prediction of the hydrodynamic force for the largest values of $U_{\max }$. For smaller exit velocities, the added mass approach largely underestimates the hydrodynamic force. The reason of these differences is very likely related to the influence of gravity, which is not included in the added mass approach. However, the measured hydrodynamic force still follows a time evolution similar to the time evolution of the acceleration, despite the fact that the magnitude of the body acceleration is much smaller than the gravity acceleration for $U_{\max }=0.2 \mathrm{~m} / \mathrm{s}$ (see figure $7 \mathrm{~b}$ ).

In figures 39 and 40, the evolution of the radius of the wetted surface measured during the water entry and exit of the cone is compared to the theoretical prediction of the radius of the wetted surface obtained from the combined Wagner-modified von Karman model. This model was proposed by Tassin et al. (2013) for the 2D case and is directly applicable to the axisymmetric case. When computing the theoretical results, we assumed that the altitude of the tip of the cone, $z$, was given by the equation $z=h(t)+\delta_{z}$ with $\delta_{z}=3 \mathrm{~mm}$ in order to compensate for the meniscus (see section 2.3), leading to $c(t)=-4\left[\delta_{z}+h(t)\right] /\left[\pi \tan \left(15^{\circ}\right)\right]$. The results obtained from the Wagner model compare remarkably well with the experimental results during the entry stage, despite the fact that the Wagner model does not take into account the gravity in the computation of the radius of the wetted surface. However, the agreement between the modified von Karman model and the experimental results is significantly less satisfactory during the exit stage. The results obtained from the model of Korobkin et al. (2017a) for $\gamma=1$ and $\gamma=2$ are also depicted in figures 39 and 40 . For these last results, the wetted surface at the beginning of the exit stage (when $\dot{h}(t)=0$ ) was set to the experimental value. The wetted surface evolutions obtained with $\gamma=1$ are very close to the experimental data at the beginning of the exit stage, but the theoretical model overestimates the wetted surface at the end of the exit stage because of the effect of gravity. Note however that Korobkin 


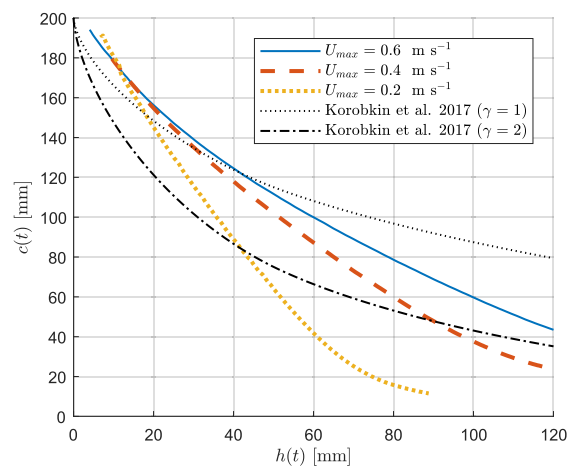

Figure 36: Comparison between the wetted surface radius predicted by the model of Korobkin et al. (2017a) and the one measured during the water exit of the disc

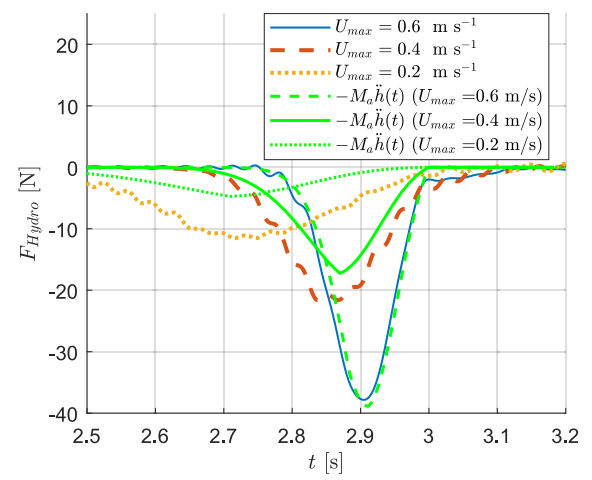

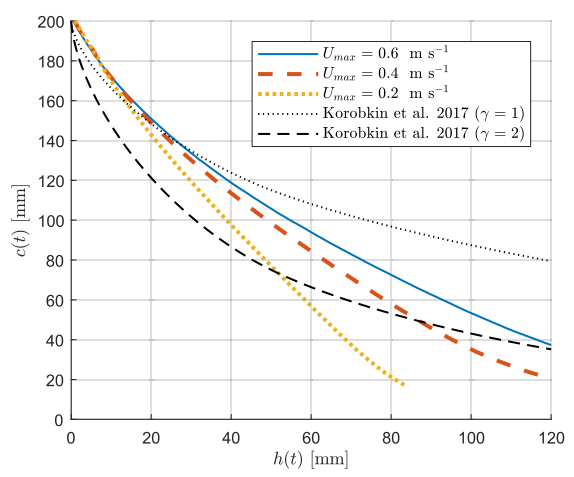

Figure 37: Comparison between the wetted surface radius predicted by the model of Korobkin et al. (2017a) and the one measured during the water exit of the cone

Figure 38: Comparison between the hydrodynamic force predicted by the linearized added mass approach and the one measured during the water exit of the disc

(2013) found that a value of $\gamma=2$ led to a better prediction of the hydrodynamic force for large values of body elevation (when compared to numerical results obtained from a CFD approach). In order to improve the linearized model so as to get a consistent prediction of the hydrodynamic force and of the wetted surface, it seems necessary to improve the hydrodynamic force/pressure formulation.

\section{Conclusion - Discussion}

In this paper, we have presented an experimental study on the water exit and entry-exit of axisymmetric bodies both flat and three-dimensional. The experimental set-up makes it possible to measure simultaneously the evolution of the wetted surface and of the hydrodynamic force. The LED edge-lighting technique proposed in Tassin et al. (2017) was employed to track the evolution of the wetted surface. We have now demonstrated the feasibility of the LED edge-lighting technique in a medium-scale wave tank with mock-ups of sizes up to $50 \mathrm{~cm}$ diameter. The accuracy of the technique was validated 


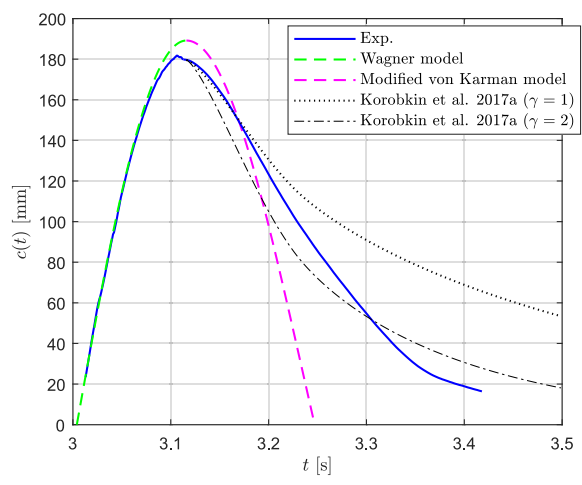

Figure 39: Evolution of the radius of the wetted area for the water entry and exit of a cone with $U_{\max }=0.57 \mathrm{~m} / \mathrm{s}$ and $c_{\max }=200 \mathrm{~mm}$

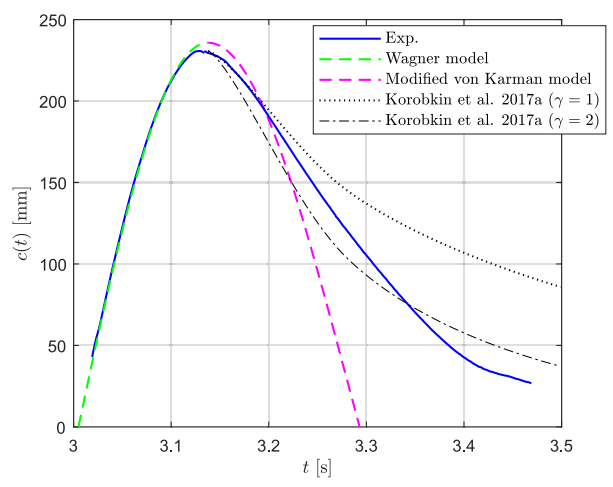

Figure 40: Evolution of the radius of the wetted area for the water entry and exit of a cone with $U_{\max }=0.6 \mathrm{~m} / \mathrm{s}$ and $c_{\max }=250 \mathrm{~mm}$

through comparisons with experiments during which a draughtboard was placed at the bottom of the tank. The LED technique makes it possible to track the contact line during the water exit of axisymmetric bodies both flat and three-dimensional. During the water entry stage of three-dimensional bodies, it is possible to observe simultaneously the contact line and the front of the jet, which is a remarkable advantage compared to the standard approach based on the use of non-transparent mock-ups.

The obtained results provide different insights on the physics of the water entry phenomenon. The experiments conducted in Froude similarity with different values of the initial radius of the wetted surface show that the similarity is verified. This indicates that viscosity and surface tension do not have a significant influence in the present experiments (except for the presence of the meniscus). Our experiments confirm that the shape of the body does not play an important role in the evolution of the contact surface during water exit. The experimental results show that the evolution of the contact surface during an exit stage which follows an entry stage is very similar to the one observed during a pure water exit experiment. It is also interesting to notice that for the experiments with the largest velocity $\left(U_{\max }=0.6 \mathrm{~m} / \mathrm{s}\right)$, the evolution of the contact line follows a similar law as the theoretical prediction of the self-similar model of Korobkin et al. (2017b).

The results of the water entry experiments have been compared to the predictions of the Wagner model. It was shown that it is possible to predict very accurately the evolution of the radius of the wetted surface with the Wagner model, despite the influence of gravity in our experiments. For the exit stage, the results obtained with the modified von Karman model are much less satisfactory. An extension of the linearized water exit model of Korobkin et al. (2017a) has been implemented in order to model the water exit of a rigid axisymmetric body with an arbitrary exit kinematics $(\ddot{h}(t) \geqslant 0)$. The theoretical results obtained from this model with $\gamma=1$ are in good agreement with the experimental results at the beginning of the exit stage (when the effect of gravity is negligible). However, the theoretical model overestimates the wetted surface when the elevation and the effect of gravity increase. It is interesting to note that the exit stage seems to be more affected by gravity than the entry stage.

An original aspect of the experimental set-up used in the present study is that the evolution of the surface of contact between the solid and the liquid is tracked using an optical method based on the use of a LED edge-lightning technique and transparent mock- 
ups. This method offers several advantages compared to the draughtboard technique or to the use of opaque mock-ups. The LED technique can be used in large and deep basins, which is less convenient with the two other techniques. With the LED edge-lighting technique, the video camera is in air whereas with opaque mock-ups it is necessary to use an underwater camera or mirrors when possible. The edge-lighting technique makes it possible to track simultaneously the contact line and the jet front during the entry stage, which is not possible with opaque mock-ups. It is most likely possible to apply the LED edge-lighting technique to mock-ups subject to a rotation (e.g. pitch motion), which would be nearly impossible with the draughtboard technique.

The LED edge-lighting technique has nevertheless a few disadvantages. In particular, the mock-up and the water have to be clean. The intensity of the light is rather low which necessitates the high-speed video camera to have a good light sensitivity. The surface of the mock-ups has to be very finely polished.

In future, we are planning to apply this technique to more complex motions including rotations and to freely moving mock-ups (e.g. sea-keeping experiments). Eventually, we would like to study wave impacts on three-dimensional shapes (promising preliminary results have been obtained with the sphere). The edge-lighting technique may be used for other applications where the tracking of the contact line and of the jet front are important (e.g. droplet impacts on solid substrates, sloshing in tanks). Additional results on the water exit of an elliptical disc and of a square plate should be published soon.

\section{Acknowledgements}

T.B. and A.T. acknowledge the technical assistance of Sébastien Chalony from Ifremer for his help in designing, preparing and conducting the experiments. T.B. and A.T. acknowledge the team members of Ifremer's wave and current flume in Boulogne-surMer for their assistance during the two experimental campaigns. The authors would like thank Dr Romain Hascoët from ENSTA Bretagne for his careful reading of the manuscript before submission. Dominique Le Roux from Ifremer contributed to the initial design of the experimental set-up. The experimental set-up was funded by Institut Carnot Ifremer-EDROME. This work was also supported by Région Bretagne (ARED funding) through the $\mathrm{PhD}$ grant of T.B..

Declaration of Interests. The authors report no conflict of interest.

\section{Appendix A. Validation of the LED edge-lighting technique for the different mock-ups}

In this appendix, we compare the results obtained with the LED edge-lighting technique and the results obtained with the draughtboard technique in order to assess the accuracy of the edge-lighting technique for the tracking of the contact line during the water exit experiments with the diffrent shapes. All the images depicted in this section were captured at a frame rate of $1000 \mathrm{fps}$. The online version of the article can be used to zoom in on small details of the images.

\section{A.1. Water exit of the circular disc}

A sequence of four images recorded during the water exit of the circular disc for different values of the elevation, $h(t)$, is plotted in figure 41. In these images, the inner illuminated ring which contracts towards the centre of the image as the elevation increases 


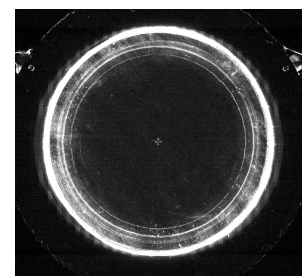

(a) $h=18.0 \mathrm{~mm}$

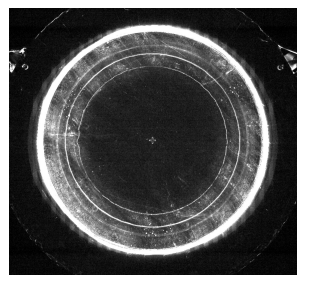

(b) $h=33.0 \mathrm{~mm}$

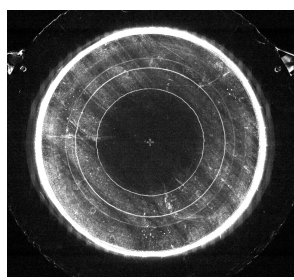

(c) $h=61.4 \mathrm{~mm}$

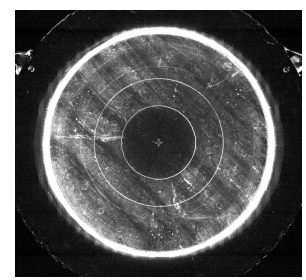

(d) $h=91.4 \mathrm{~mm}$

Figure 41: Sequence of images recorded during the water exit of the $200 \mathrm{~mm}$ radius circular disc with the LED edge-lighting technique for $U_{\max }=0.6 \mathrm{~m} / \mathrm{s}$

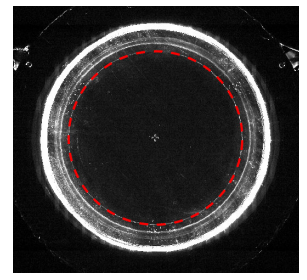

(a) $h=18.0 \mathrm{~mm}$

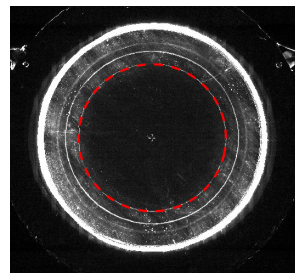

(b) $h=33.0 \mathrm{~mm}$

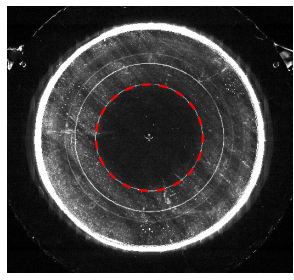

(c) $h=61.4 \mathrm{~mm}$

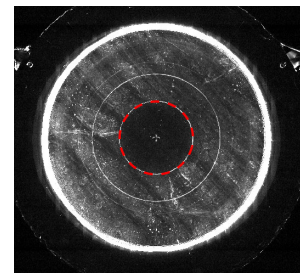

(d) $h=91.4 \mathrm{~mm}$

Figure 42: Detection of the contact line (red dashed line) from the images obtained with the LED edge-lighting technique during the water exit of the $200 \mathrm{~mm}$ radius circular disc with the LED edge-lighting technique for $U_{\max }=0.6 \mathrm{~m} / \mathrm{s}$

corresponds to the contact line. The outer concentric rings are attributed to the direct diffusion of light due to the unperfect polishing of the sides of the mock-up and to undesired specular reflections of the light source on the deformed free surface. Similarly to the results presented in section 3.2 for the water entry and exit of the cone, the contact line geometry is extracted using a contour detection algorithm based on the method of Taubin (1991) and assuming that the shape of the contact line is elliptic. The performance of the contour tracking algorithm is illustrated in figure 42 where the detected contours and the original images (from figure 41) are superimposed.

In order to verify that the inner ring corresponds to the contour of the wetted surface (the contact line), the same experiments were conducted with a draughtboard laying at the bottom of the tank (approximately $2 \mathrm{~m}$ below the free surface). With this technique, the region over which the water is in contact with the mock-up corresponds to the region over which the draughtboard appears undistorded (see figure 43). The red dashed line which is superimposed to the images obtained with the draughtboard technique in figure 43 corresponds to the contour extracted from figure 42 . One can observe that the region over which the draughtboard remains undistorded is perfectly circumscribed by the contour extracted from figure 42. This comparisons demonstrates the accuracy of the LED edge-lighting technique in the case of the circular disc.

\section{A.2. Water exit of the cone}

The same verification was performed for the cone. A sequence of images obtained during the water exit of the cone with an initial radius of the wetted surface $c_{0}=200$ $\mathrm{mm}$ is depicted in figure 44. Similarly to the images obtained during the water exit of the circular disc, one can observe an inner illuminated ring which contracts towards the center of the image. Note that the tip of the cone coincides with the bright dot which is visible at the centre of the images. The contours extracted from these images are plotted 


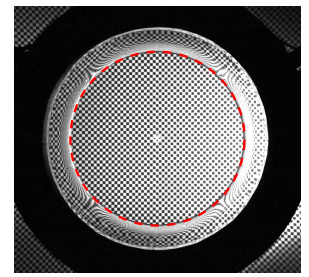

(a) $h=18.0 \mathrm{~mm}$

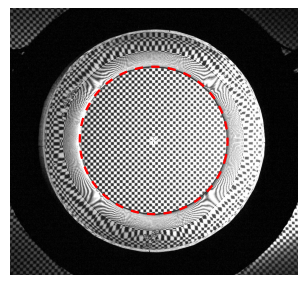

(b) $h=33.0 \mathrm{~mm}$

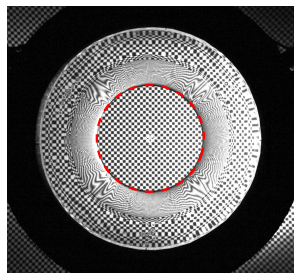

(c) $h=61.4 \mathrm{~mm}$

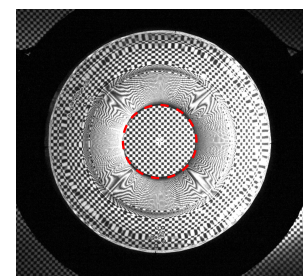

(d) $h=91.4 \mathrm{~mm}$

Figure 43: Comparison between the contact line obtained with the LED edge lighting technique (red dashed line) and the images obtained with the draughtboard during the water exit of the $200 \mathrm{~mm}$ radius circular disc for $U_{\max }=0.6 \mathrm{~m} / \mathrm{s}$

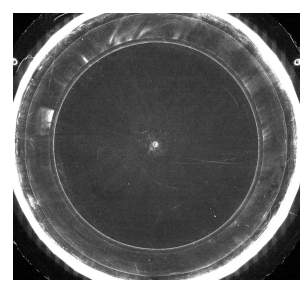

(a) $h=2.4 \mathrm{~mm}$

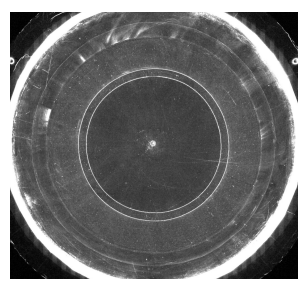

(b) $h=31.5 \mathrm{~mm}$

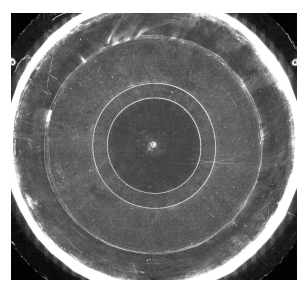

(c) $h=60.8 \mathrm{~mm}$

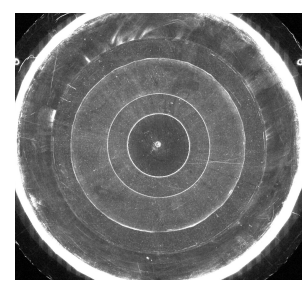

(d) $h=90.8 \mathrm{~mm}$

Figure 44: Sequence of images recorded during the water exit of the cone with an initial value of the radius of the wetted surface $c_{0}=200 \mathrm{~mm}$ for $U_{\max }=0.6 \mathrm{~m} / \mathrm{s}$

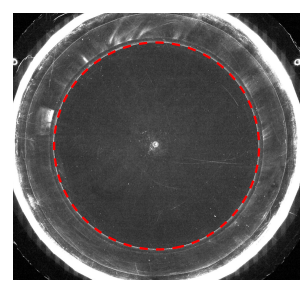

(a) $h=2.4 \mathrm{~mm}$

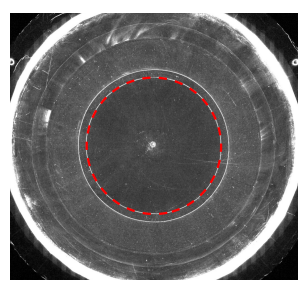

(b) $h=31.5 \mathrm{~mm}$

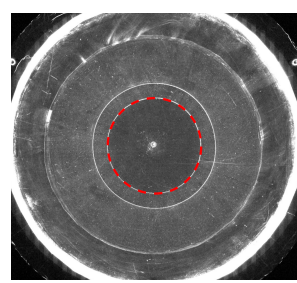

(c) $h=60.8 \mathrm{~mm}$

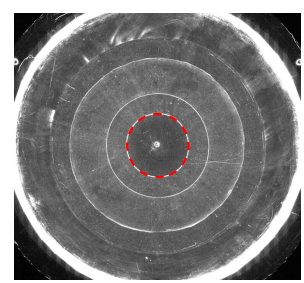

(d) $h=90.8 \mathrm{~mm}$

Figure 45: Detection of the contact line (red dashed line) from the images obtained with the LED edge-lighting technique during the water exit of the cone initially in contact with the water on a surface of radius $c_{0}=200 \mathrm{~mm}$ for $U_{\max }=0.6 \mathrm{~m} / \mathrm{s}$

in figure 45 and superimposed to the images obtained with the draughtboard technique during the water exit of the cone in figure 46. One observes that the contour illuminated with the LED edge-lighting technique closely corresponds to the contour of the region over which the draughtboard is distorted only by the conical shape of the mock-up.

\section{A.3. Water exit of the sphere}

A sequence of four images obtained during the water exit of the sphere with the LED edge-lighting technique is plotted in figure 47. Note that the position of the axis of symmetry is marked by a bright dot at the centre of the mock-up. The contours extracted from these images are superimposed to the original images in figure 48 and to the images obtained with the draughtboard technique in figure 49. One can appreciate the close correlation between the contact line illuminated with the LED edge-lighting technique and the contour of the wetted surface obtained with the draughtboard technique. It 


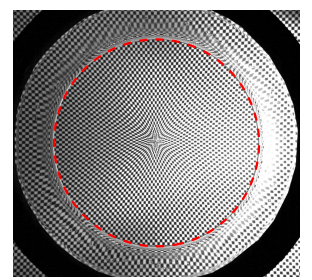

(a) $h=2.4 \mathrm{~mm}$

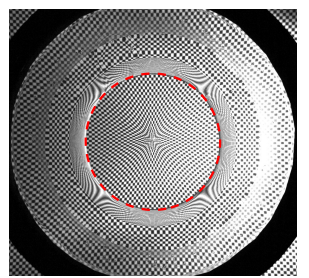

(b) $h=31.5 \mathrm{~mm}$

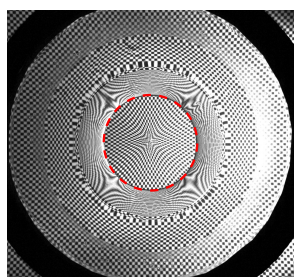

(c) $h=60.8 \mathrm{~mm}$

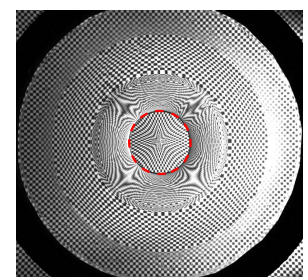

(d) $h=90.8 \mathrm{~mm}$

Figure 46: Comparison between the contact line obtained with the LED edge-lighting technique (red dashed line) and the images obtained with the draughtboard technique during the water exit of the cone initially in contact with the water on a surface of radius $c_{0}=200 \mathrm{~mm}$ for $U_{\max }=0.6 \mathrm{~m} / \mathrm{s}$

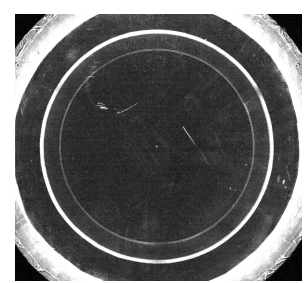

(a) $h=0.3 \mathrm{~mm}$

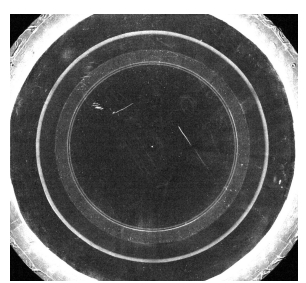

(b) $h=10.9 \mathrm{~mm}$

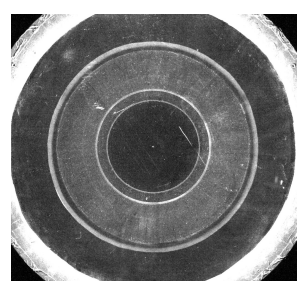

(c) $h=60.8 \mathrm{~mm}$

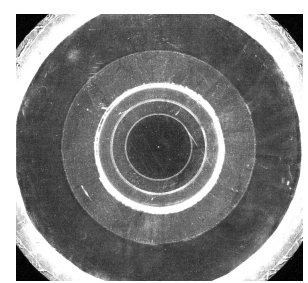

(d) $h=90.8 \mathrm{~mm}$

Figure 47: Sequence of images recorded during the water exit of the sphere with an initial value of the radius of the wetted surface $c_{0}=200 \mathrm{~mm}$ for $U_{\max }=0.6 \mathrm{~m} / \mathrm{s}$

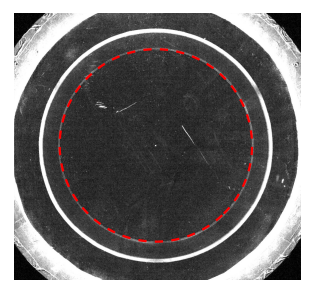

(a) $h=0.3 \mathrm{~mm}$

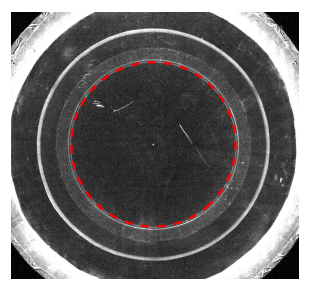

(b) $h=10.9 \mathrm{~mm}$

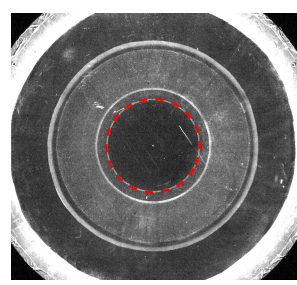

(c) $h=60.8 \mathrm{~mm}$

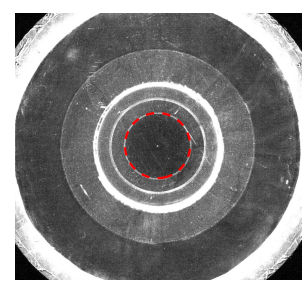

(d) $h=90.8 \mathrm{~mm}$

Figure 48: Detection of the contact line (red dashed line) from the images obtained with the LED edge-lighting technique during the water exit of a sphere initially in contact with the water on a surface of radius $c_{0}=200 \mathrm{~mm}$ for $U_{\max }=0.6 \mathrm{~m} / \mathrm{s}$

should be noted that in the case of the sphere, the tracking of the luminous contour is made a bit more difficult at the beginning of the exit because of the presence of a secondary luminous ring very close to the contour of interest and which may be mistaken for the contact line by the tracking algorithm. The results obtained with the LED edgelighting technique remain nevertheless very accurate in the case of the sphere. 
Experimental investigation of the water entry and/or exit of axisymmetric bodies 31

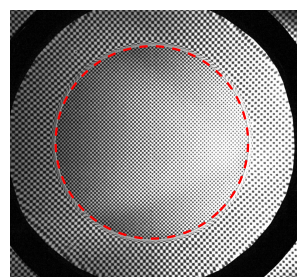

(a) $h=0.3 \mathrm{~mm}$

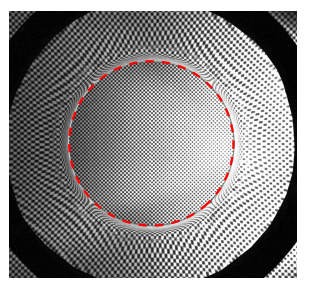

(b) $h=10.9 \mathrm{~mm}$

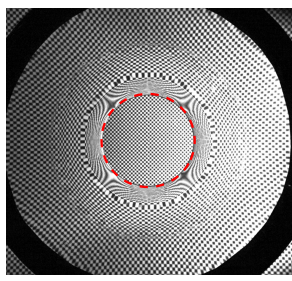

(c) $h=60.8 \mathrm{~mm}$

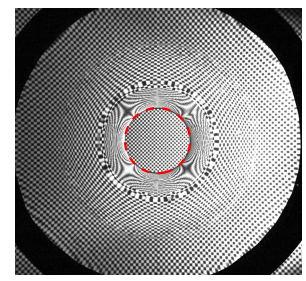

(d) $h=90.8 \mathrm{~mm}$

Figure 49: Comparison between the contact line obtained with the LED edge-lighting technique (red dashed line) and the images obtained with the draughtboard during the water exit of a sphere initially in contact with the water on a surface of radius $c_{0}=200$ $\mathrm{mm}$ for $U_{\max }=0.6 \mathrm{~m} / \mathrm{s}$ 


\section{REFERENCES}

BaArholm, R. \& Faltinsen, O. M. 2004 Wave impact underneath horizontal decks. Journal of Marine Science Technology 9, 1-13.

Bensch, L., Shigunov, V., Beuck, G. \& Söding, H. 2001 Planned ditching simulation of a transport airplane. In KRASH USERS SEMINAR. 7-10 January 2001, Phoenix, Arizona.

Duez, C., Ybert, C., Barentin, C., Cottin-Bizonne, C. \& Bocquet, L. 2008 Dynamics of fakir liquids: from slip to splash. Journal of Adhesion Science and Technology 22, 335-351.

El Malki Alaoui, A., Nême, A., Tassin, A. \& Jacques, N. 2012 Experimental study of slamming coefficients during vertical water entry of axisymmetric rigid shapes at constant speeds. Applied Ocean Research 37, 183-197.

Halbout, S. 2011 Contribution à l'étude des interactions fluide-structure lors de l'impact hydrodynamique avec vitesse d'avance d'un système de flottabilité d'hélicoptère (in french). PhD thesis, Université d'Aix-Marseille, France.

Iafrati, A. \& Grizzi, S. 2019 Cavitation and ventilation modalities during ditching. Physics of Fluids 31 (5), 052101, arXiv: https://doi.org/10.1063/1.5092559.

KAPLAN, P. 1987 Analysis and prediction of flat bottom slamming impact of advanced marine vehicles in waves. International Shipbuilding Progress 34 (391), 44-53.

Korobkin, AA 2007 Second-order wagner theory of wave impact. Journal of Engineering Mathematics 58 (1-4), 121-139.

Korobkin, A., Khabakhpasheva, T. \& Maki, K. $2017 a$ Hydrodynamic forces in water exit problems. Journal of Fluids and Structures 69, 16-33.

Korobkin, A, Khabakhpasheva, T. \& Rodríguez-Rodríguez, J $2017 b$ Initial stage of plate lifting from a water surface. Journal of Engineering Mathematics pp. 1-14.

Korobkin, A. A. 2013 A linearized model of water exit. Journal of Fluid Mechanics 737, $368-386$.

Korobkin, A. A. \& Scolan, Y.-M. 2006 Three-dimensional theory of water impact. Part 2. Linearized Wagner problem. Journal of Fluid Mechanics 549, 343-373.

Panciroli, R., Abrate, S., Minak, G. \& Zucchelli, A. 2012 Hydroelasticity in waterentry problems: Comparison between experimental and SPH results. Composite Structures $94(2), 532-539$.

Piro, Dominic J. \& Maki, Kevin J. 2013a An adaptive interface compression method for water entry and exit. Tech. Rep. 2013-350. University of Michigan Department of Naval Architecture and Marine Engineering.

Piro, D. J. \& MAKI, K. J. $2013 b$ Hydroelastic analysis of bodies that enter and exit water. Journal of Fluids and Structures 37, 134-150.

Reinhard, M. 2013 Free elastic plate impact into water. PhD thesis, University of East Anglia, UK.

Reinhard, M., Korobkin, A. A. \& Cooker, M. J. 2012 The bounce of a blunt body from a water surface at high horizontal speed. In $27^{\text {th }}$ International Workshop on Water Waves and Floating Bodies. 22-25 April 2012, Copenhagen, Denmark.

Reis, Pedro M., Jung, Sunghwan, Aristoff, Jeffrey M. \& Stocker, Roman 2010 How cats lap: Water uptake by felis catus. Science 330, 1231-1234.

Scolan, Y.-M., Remy, F. \& Thibault, B. 2006 Impact of three-dimensional standing waves on a flat horizontal plate. In $21^{\text {st }}$ International Workshop on Water Waves and Floating Bodies. 2-5 April 2006, Loughborough, U.K.

Semenov, Y. A. \& Yoon, B.-S. 2009 Onset of flow separation for the oblique water impact of a wedge. Physics of Fluids 21, 112103.

Shams, A., Zhao, S. \& Porfiri, M. 2017 Hydroelastic slamming of flexible wedges: Modeling and experiments from water entry to exit. Physics of Fluids 29 (3), 037107.

Sun, Hui \& Helmers, Jens B 2015 Slamming loads on a wedge elastically suspended on a marine structure. In ASME 2015 34th International Conference on Ocean, Offshore and Arctic Engineering. American Society of Mechanical Engineers.

Tang, Z., von Gioi, R.G., Monasse, P. \& Morel, J.-M 2017 A precision analysis of camera distortion models. IEEE transactions on Image Processing 26, 2694-2704.

Tassin, A., Breton, T., Forest, B., Ohana, J., Chalony, S., Le Roux, D. \& Tancray, A. 2017 Visualization of the contact line during the water exit of flat plates. Experiments in Fluids 58 (8), 104. 
Experimental investigation of the water entry and/or exit of axisymmetric bodies 33

Tassin, A., Jacques, N., El Malki Alaoui, A., Nême, A. \& Leblé, B. 2012 Hydrodynamic loads during water impact of three-dimensional solids: Modelling and experiments. Journal of Fluids and Structures 28, 211-231.

Tassin, A., Piro, D. J., Korobkin, A. A., Maki, K. J. \& Cooker, M. J. 2013 Twodimensional water entry and exit of a body whose shape varies in time. Journal of Fluids and Structures 40, 317-336.

TAubin, G. 1991 Estimation of planar curves, surfaces, and nonplanar space curves defined by implicit equations with applications to edge and range image segmentation. IEEE Transactions on Pattern Analysis and Machine Intelligence 13 (11), 1115-1138.

Vega-Martínez, P, Rodríguez-Rodríguez, J, Khabakhpasheva, T. \& Korobkin, A 2019 Hydro-elastic effects during the fast lifting of a disc from a water surface. Journal of Fluid Mechanics 869, 726-751.

Wagner, H. 1931 Landing of seaplanes. NACA Technical Memorandum 622 pp. 1-23.

WAGner, H. 1932 Über Stoß- und Gleitvorgänge an der Oberfläche von Flüssigkeiten. ZAMM 12, 193-215, (in German). 